\title{
Viabilist and Tychastic Approaches to Guaranteed ALM Problem
}

\author{
Jean-Pierre Aubin, Luxi Chen, \\ Olivier Dordan and Patrick Saint-Pierre
}

version du 10 décembre 2010

To Alain Bensoussan,

in friendly remembrance of this fruitful tychastic encounter of the summer of 1969 in Madison, which has contributed to my decision to return to France to found with him CEREMADE (Centre for Research in Mathematics of the Decision) of the University Paris-Dauphine, this contribution which revisits forty years later his pioneering work on impulse management and finance market to which he initiated me.

Jean-Pierre Aubin

\section{Introduction}

This study reconsiders the problem of hedging a liability by a portfolio made of a riskless asset and an underlying (underlying). This is a dynamical mechanism ensuring that, at each date, the value of the portfolio is "always" exceeding liabilities.

This is a very simple problem of viability, which has prompted the emergence of concepts and mathematical and algorithmic results gathered under the name of "viability theory". It is quite natural to use them for determine ALM cushion management mechanisms as, for example, the CPPI (Constant Proportional Portfolio Insurance) that Merton discovered as optimal strategy for maximizing a utility function. In their paper [39, Boulier \& Kanniganti], the authors describe it in the following way : [...] An alternative approach [...] is based on the following two ideas : first, the portfolio is always maintained above a certain minimum level called the floor, the difference or the "surplus" being called the "cushion"- the floor is assumed to grow at a fixed rate (for example, at the risk-less rate of interest) such that at the maturity of the fund, it is at least equal to the guaranteed amount; second, the exposure to the market at any moment is determined as a (non-decreasing) function of the cushion, usually a constant multiple of the cushion. [...] The CPPI is a technique easy to understand and implement, and independent of time. [...] There is a small risk of the portfolio crashing through the floor in between two rebalancements, as happened with some assured portfolios 
during the 1987 crash. In such a case, it is impossible even to meet the guarantee. Therefore, one objective of management might be to minimize this possibility.

The aim of viability theory is not to minimize this possibility, but, simply, to eradicate it.

For another problem is hidden under the "always" contained in the statement of the problem, which conceals an uncertainty against which we must be guarded. Probability and stochastic theories are naturally available to us for addressing this issue. However, the management rules derived from CPPI do not yet meet the "I" of Insurance, as there are realizations of future prices for which this viability property is violated.

This observation alone justifies a reexamination of possibilities for offering other (mathematical) viewpoints to translate the many facets of the polysemous concept of uncertainty.

The failure of the CPPI rule to guarantee that the floor is never pierced by the value of the portfolio questions the ability of probability and stochastic theory to be the only possible translation of uncertainty, and suggests other ones, such as tychastic, contingent and impulse uncertainty to approach the asset-liability management issues.

It starts from the observation that we know the variables on which bears uncertainty : the future risky returns unknown at the investment date when the initial investment must be computed. We also know all the potential (contingent) ways to manage portfolio by determining the exposure (the risky component) of the portfolio. We know from experience that it is possible to restore viability when the liability is reached, by borrowing, instantly (through an impulse), an authorized loan to pay off the forthcoming ruin.

Economic theory is dedicated to the analysis and the computation of supply and demand adjustment laws, among which the Walras tâtonnement, in the hope of explaining the mechanisms of price formation (see for instance [3, Aubin]). In the last analysis, the choice of the prices is made by the invisible hand of the "Market", the new deity in which many economists and investors believe. Their worshippers may not realize that He may listen to their prayers, but that $\mathrm{He}$ is reacting to their actions in a carefully hidden way. Unfortunately, economic theory does not provide explicit or computable pricing mechanisms of assets and underlying, the commodities of the financial markets constituting portfolios.

In most financial scenarios, investors take into account their ignorance of the pricing mechanism. They assume instead that prices evolve under uncertainty, and that they can master this uncertainty. They still share the belief that the "Market knows best" how to regulate the prices, above all without human or political regulations. The question became to known how to master this uncertainty. For that, many of them trade the Adam Smith invisible hand against a Brownian movement, since it seems that this unfortunate hand is shaking like a particle on the surface of a liquid. It should then be enough to assume average returns and volatilities to be known for managing portfolios. 
We accept the same attitude, but we exchange the Adam Smith invisible hand against tychastic uncertainty instead of a stochastic one on the formation of asset prices for deriving management rules of the portfolio satisfying the required scarcity constraint : the value of the portfolio is always larger or equal to the liabilities.

We then propose to use three other aspects of uncertainty (among known and still unknown ones).

The first, called tychastic uncertainty, considers the set of risky returns as a "tychastic reservoir" in which returns appear unexpectedly, examples of tyche ("chance" in Greek), over which the investor has no control. The problem is no longer to assess the probability of risky returns realizations, but to determine the subset in which they can emerge. Prediction models or extrapolation techniques no longer consist in determining trends and volatilities, but in the tychastic case, the lower bounds of risky returns defining the future tychastic reservoir. By the way, the available information provided by brokerage firms (lower and upper bounds of prices, returns the riskless asset) allows us to extrapolate them and to forecast the future returns of the underlying.

The second, referred to as contingent uncertainty, is described by a "contingent reservoir" composed of portfolio exposures in which the investor can draw upon to manage the portfolio in a contingent manner. Management rules are time series of exposures, that is to say, of allocations of the values of the portfolio to risky and not risky components, that the investor has the freedom to choose : in our approach, the management rule is the solution to the problem rather than a given datum.

The third, called impulse uncertainty, involves an "impulse reservoir" composed of a set of reset feedbacks. Defined on the subset (trap) on which viability is at stake, reset feedbacks remedy immediately, with infinite velocity (impulse) for restoring the state of the system outside the trap. Very often, the trap is a subset of the boundary of the environment, but not always. In the example we are concerned with, the trap is the floor. Given a flow of loans authorized in advance, the reset map adds a cushion to the value of the floor, which is regarded as an authorized loan he is entitle to use. The "impulse management" uses contingent feedback as long as the value of the portfolio is strictly above the floor and switches to the reset feedback when the portfolio hits the floor to start again with a higher value.

This impulse management method avoids prediction of disasters, but offers opportunities to recover from them when they occur. Instead of seeking an insurance from a tychastic reservoir assumed to be known or predicted (predictive approach), the impulse approach allows the investor to borrow authorized loans when the value reaches the floor.

It seems that the strategy to build a reservoir of reset feedbacks is used by living beings to adapt to their environment before the primates that we are unwisely seek to predict their future while being quite unable to do so. The impulse approach announces the death of the soothsayers and the emergence of demiurges remedying unforeseen disasters, because most 
often unpredictable.

These are two approaches which are presented in this study.

This study uses these three aspects of uncertainty. Contingent uncertainty to manage the evolution of the value of the portfolio as long as viability is not at stake, tychastic uncertainty that disturbs without apparent reason and without any statistical regularity this evolution, whereas impulse uncertainty uses available reset feedbacks to restore viability.

In both cases (predictive and impulse approaches), we are looking for a management rule of portfolio exposures providing a value of the portfolio to be, at any future date, larger than or equal to the liability whatever the risky returns. These two "worst case" approaches make stress tests unuseful and obsolete.

The first section of this study states the problem of hedging a liability by an asset portfolio, the second details the different types of uncertainty. The predictive and impulse approaches are exposed in the third section, illustrated in the fourth section with examples derived from algorithms. The flow charts are presented in section five. The sixth section presents the minimum concepts of viability theory for controlled tychastic systems that define the problem of asset-liability cushion management by methods which inherit the mathematical and algorithmical results of viability theory. The study concludes with a comparison between the viability and tychastic stochastic approach due to Halim Doss, who concluded that stochastic viability is a special case of tychastic viability.

\section{Asset-Liability Management : the Insurance Pro- blem}

\subsection{Insuring Liabilities}

The liability flow to insure is described as a constraint floor not to be "pierced". The floor is defined by a function $\mathbf{k}: t \geq 0 \mapsto \mathbf{k}(t) \geq 0$.

The capital to guarantee (at exercise time) is the final value $\kappa(T)$ the floor to the exercise date $T$.

Example : "variable annuities". They are described by a floor defined in the following way : at each date $t$, the floor associated with an objective of cash flow is defined by

1. a floor return $\rho(t)$

2. a schedule $\kappa\left(t_{n}\right)(n=0, \ldots, N)$ of expenditures or incomes at dates $t_{n}$

The floor is governed by an (impulse) differential equation. It is no longer continuous, but punctuated by "jumps" at the dates of the schedule (the floor is supposed to be only lower semicontinuous). 


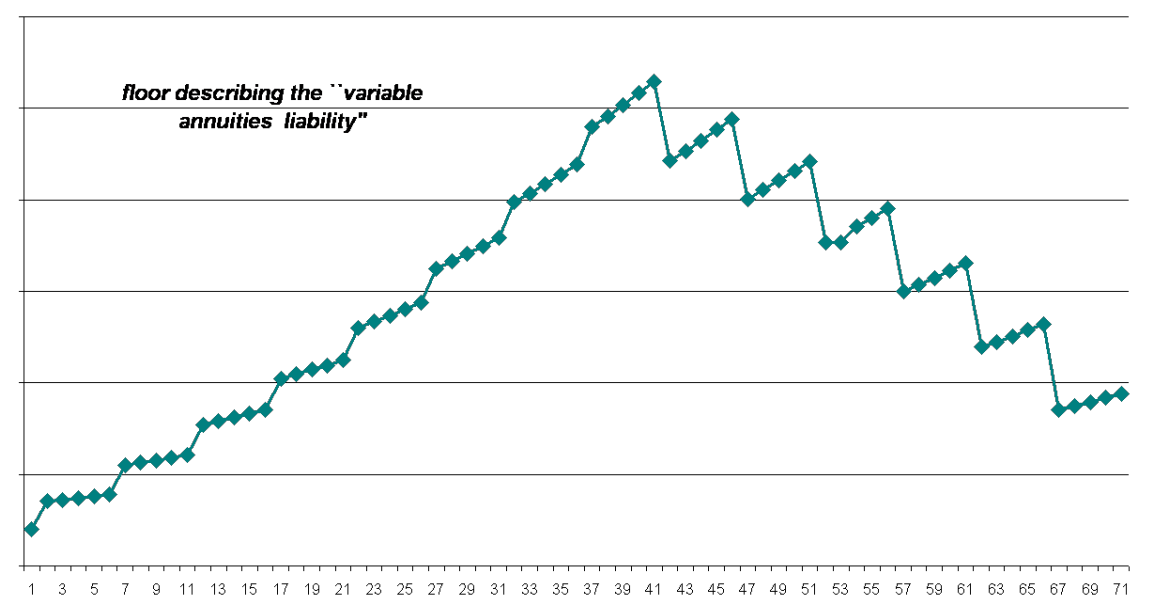

1 "Variable Annuities" Floor In this example of a pension plan (which must pay benefits to pensioners on a fixed date), the figure shows the floor $\mathbf{k}(t)$ describing the "variable annuities liability" over a period of seventy-five years with provision of funds set every five years during the forty years investment period and payment of benefits every five years during the last thirty-five dates.

In this study, the floor is assumed to depend only on time. The case when it depends on transaction values (for taking into account brokerage fees, for instances) or on the price of the asset can be studied in the same way. See for instance [49, Planchet \&Therond] and [50, Planchet, Kamega A. \&Therond].

Remark : portfolio replicating options - They have been studied in the framework of viability theory, but in a context different from the VPPI : it is not required that the value of the portfolio is always above the floor (viability property), but only at the first time when the value of the portfolio reaches the floor (capturability property) after which the option is exercised. In this case, the floor depends on the asset prices. For European option, for instance, $\mathbf{k}(T, S):=\max (0, S-K)$ (where $K$ denotes the exercise price) and $\mathbf{k}(t, S)=0$ for $0 \leq t<T$, whereas, for American options, $\mathbf{k}(t, S):=\max (0, S-S-K)$ for $0 \leq t \leq T$. (See [18, Aubin, Pujal \& Saint-Pierre] and [19, Aubin \& Saint-Pierre] in the tychastic case and, among many other contributions, [21, 22, 23, 24, 25, 26, Bensoussan], [27, 29, 30, 31, Bensoussan, Crouhy \& Galai], [32, 33, Bensoussan \& Julien], [34, Bensoussan, Keppo \& Sethi], etc.).

\subsection{Assets portfolios and their exposure}

The question is "to hedge" the liability by a portfolio made of of a riskless asset and of an underlying (a "risky asset", in many examples). 
Given different fees depending on the value of the portfolio (brokerage, management, commissions, etc.), the net value of the portfolio is obtained after deducting these fees. The (net) cushion is the difference between the (net) value of the portfolio and the floor.

Denote by 0 the investment date, by $T>0$ the exercise time (or, the term, the horizon, etc.), by $t \geq 0$ the current (or spot) time and by $T-t \geq 0$ the time to maturity. We set :

$$
\left\{\begin{array}{l}
S^{0}(t) \text { the price of the riskless asset, } \\
S(t) \quad \text { the price of the underlying, } \\
R^{0}(t)=\frac{\frac{d}{d t} S^{0}(t)}{S^{0}(t)} \text { the return of the riskless asset, } \\
R(t)=\frac{\frac{d}{d t} S(t)}{S(t)} \text { the return of the underlying, } \\
P^{0}(t) \text { the number of shares of the riskless asset, } \\
P(t) \text { the number of shares of the underlying, } \\
W(t)=P^{0}(t) S^{0}(t)+P(t) S(t) \text { the value of the portfolio, } \\
E^{0}(t)=P^{0}(t) S^{0}(t) \text { the liquid component of the portfolio, } \\
E(t)=P(t) S(t) \text { the exposure (risky component) of the portfolio, } \\
V(t)=S^{0}(t) \frac{d}{d t} P^{0}(t)+S(t) \frac{d}{d t} P(t) \text { the transaction value of the portfolio, } \\
C\left(t_{n}\right)=\text { the coupon (impulsive cash flow) } \\
\text { at dates } \tau_{n} \text { of the coupon schedule. }
\end{array}\right.
$$

The value of the portfolio and its exposure summarize the information for deriving relevant properties in the portfolio. It follows that the formula

$$
\forall t \in[0, T], \quad W^{\prime}(t)=R^{0}(t) W(t)+E(t)\left(R(t)-R^{0}(t)\right)+V(t)
$$

describes the dynamic evolution of the portfolio controlled by the exposures $E(t)$ and transaction values $V(t)$ and dependent on the returns $R(t)$ of the underlying (tyches) unknown at investment date.

Remark - The constraints on transaction values are defined by the upper bounds $V^{\sharp}(t) \geq 0$ and lower bounds $-V^{b}(t) \leq 0$ on transaction values :

$$
\forall t \in[0, T], \quad V(t) \in\left[-V^{b}(t), V^{\sharp}(t)\right]
$$

The value of the portfolio can be depleted by various fees and commissions. The net value of the portfolio is the value after deduction of fees. The usual fees are

- management fee portfolio proportional to the cushions : $\delta(W(t)-\mathbf{k}(t))$, where $0 \leq \delta<$ 1

- brokerage fee measured by a function of the transaction value satisfying $\gamma(0)=0$.

Hypothesis : To simplify the presentation, we assume in this study that the management of the portfolio is self-financed in the sense that $\forall t \in[0, T], \quad V^{\sharp}(t)=V^{b}(t)=0$. 


\subsection{Portfolio insurance : a viability problem}

To insure or guarantee a liability by asset-liability management can be formulated in the following way : to design a management rule of the portfolio that guarantees (or insures) that the net value of the portfolio is always higher than the liability (i.e., above the floor).

$$
\forall t \in[0, T], W(t) \geq \mathbf{k}(t) \text { and } W(T)=\mathbf{k}(T)
$$

Given the known liabilities at each date, the problem is to "dynamically manage the assets" in terms of

1. the dynamics governing the evolution of the portfolio based on underlying returns (and transaction values when the portfolio is not self-financed) ;

2. various constraints characterizing the "financial product".

Despite the simplicity of this formulation, taking into account these constraints poses "viability issues". Indeed, it is expressed by stating that

$$
\forall t \in[0, T], \quad(t, W(t)) \in \mathcal{E} p(\mathbf{k}) \text { and }(T, W(T)) \in \operatorname{Graph}((\mathbf{k})
$$

where the epigraph $\mathcal{E} p(\mathbf{k})$ of the function $\mathbf{k}$ is defined by

$$
\mathcal{E} p(\mathbf{k}):=\{(t, W) \text { such that } W \geq \mathbf{k}(t)\}
$$

As a result, the hedging problem of a liability amounts to looking for evolutions $t \mapsto$ $(t, W(t))$ "viable in the epigraph" $\mathcal{E} p(\mathbf{k})$ of the function $\mathbf{k}$ in the sense that

$$
\forall t \in[0, T], \quad(t, W(t)) \in \mathcal{E} p(\mathbf{k})
$$

Instead of handling functions as in classical analysis, viability theory manipulates subsets as in set-valued analysis (see [13, Aubin \& Frankowska] or [51, Rockafellar \& Wets] for instance), and, in particular, graphs of maps and epigraphs of real-valued functions.

The study of dynamics under viability (or state) constraints is the purpose of viability theory which gathers the concepts and mathematical and algorithmic results addressing this issue (see the "viability survival kit", Section 6. p. 27, and, for a general exposition, 46, 47, Frankowska], [2, 3, Aubin] and [7, Aubin, Bayen \& Saint-Pierre].

\section{Uncertainties}

We just mention few facts on too well known stochastic uncertainty, we next proceed to comment the much less familiar three aspects of uncertainty : tychastic uncertainty (see Section 2.2 , p. 99, contingent uncertainty (see Section 2.4. p. 10 and impulse uncertainty (see Section 3.2, p. 16. 
The word stochastic refers to the Greek stokhastikos designating the draw of the rulers of the Athenian democracy. It is related to conjectures that it comes from the Latin word conjecturare.

We borrow from Charles Peirce the use of tychastic evolution he introduced in 1893 in an article nicely entitled Evolutionary Love for describing the evolution of a system dependent on tyches arising without any statistical regularity : Three modes of evolution have thus been brought before us : evolution by fortuitous variation, evolution by mechanical necessity, and evolution by creative love. We may term them tychastic evolution, or tychasm, anancastic evolution, or anancasm, and agapastic evolution, or agapasm." In this paper, Peirce associates the concept of anancastic evolution with the Greek concept of necessity, ananke, anticipating the "chance and necessity" framework that motivated viability theory.

The word tychastic comes from the Greek tyche, meaning luck, represented by the goddess Tyche, daughter of Oceanus and Tethys, whose goal was to disrupt the course of events either for good or for bad. Tyche became "Fortuna" in Latin, "rizikon" in Byzantine Greek, "rizq" in Arabic (with a positive connotation in these three cases). "Disturbances" or "perturbations" of robust control theory are examples of tyches among others (see for instance [28, Bensoussan \& Bernhard]). Fortune fortuitously left its role to randomness, originating in the French "randon", from the verb "randir", sharing the same root than the English "to run" and the German rennen. When running too fast, one looses the control of himself, the race becomes a poor "random walk", bumping over scandala (stones scattered on the way) and falling down, cadere in Latin, a matter of chance since it is the etymology of this word. Hazard was imported by William of Tyre from the crusades from Palestine castle named after a dice game, az zahr. Now dice, in Latin, is alea, famed after Julius Caesar's alea jacta est, which was actually thrown out the English language : chance and hazard took in this language the meaning of danger, itself from Latin dominarium. Being used in probability, the word random had to yield the way to tyche for describing evolutions without statistical regularity prone to extreme events.

Zhu Xi (1130-1200), 朱 喜 one of the most important unorthodox neo-Confucian of the Song dynasty, suggested that "if you want to treat everything, and as changes are infinite, it is difficult to predict, it must, according to circumstances, react to changes (literally, "follow, opportunity, reaction, change), instead of a priori action.

The four ideograms follow, opportunity, reaction, change

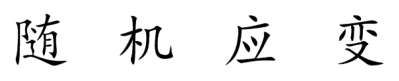

are combined to express in Chinese :

1. by the first half, “follow, opportunity", 随 机, the concept of randomness or stochasticity, 
2. while by the second half, “reaction, change", 应 变, translates the concept of tychasticity (according to Shi Shuzhong)

3. and “no, necessary”, 未 定, translates contingent.

The history of the words in different languages for taking into account the many facets of uncertainty was certainly not random, but in the context of a fascinating epistemological evolution (see for instance [6, Aubin]).

In this study, uncertainty relates to the future underlying returns unknown at decision date, which play the role of tyche.

\subsection{Stochastic Uncertainty}

Stochastic uncertainty on the returns is described by a space $\Omega$, a filtration $\mathcal{F}$, a Brownian process $W(t)$, a drift $\gamma(x)$ and a volatility $\sigma(x): d R(t)=\gamma(x(t)) d t+\sigma(x(t)) d W(t)$.

1. The random events are not explicitly identified. The set $\Omega$ is not described explicitly (one can always choose the space of all evolutions). Only the drift and volatility are assumed to be explicitly known;

2. Stochastic uncertainty does not study the "package of evolutions" (depending on $\omega \in$ $\Omega$ ), but functionals over this package, such as the different moments and their statistical consequences (averages, variance, etc.) used as evaluation of risk;

3. Required properties are valid for "almost all" constant $\omega$.

We quote a few references among so many ones : [20, Benaim \& El Karoui], [38, Bouchaud], [48, Neftci], and, in the discrete case, [55, Zabczyk].

\subsection{Tychastic Uncertainty}

In this study, tyches are returns of underlyings on which the investor has no influence. The uncertainty is described by the tychastic map defined by

$$
\mathcal{R}(t):=\left\{R \in \mathbb{R} \text { such that } R \geq R^{\mathrm{b}}(t)\right\}
$$

where $R^{b}(t)$ are the lower bounds on returns (instead of assuming that $d R(t)=\gamma(x(t)) d t+$ $\sigma(x(t)) d W(t))$.

1. Tyche are identified (returns of the underlying, for example) which can then be used in dynamic management systems when they are actually observed and known at each date during the evolution ;

2. For this reason, the results are computed in the worst case (eradication of risk instead of its statistical evaluation);

3. required properties are valid for "all" evolutions of tyches $t \mapsto R(t) \in \mathcal{R}(t)$. 


\subsection{Nature and Measures of Insurance}

In the stochastic case, the risk is "measured" by real numbers through statistical evaluation (values at risk, for example). These numbers are abstractions, which differ according to the methods and techniques used.

If the tychastic case (see Section 6.5. p. 33), the risk is "measured" by numbers, providing at each date $t$ the amount $W^{\odot}(t)$, the "minimum guaranteed investment", ensuring that the floor will never be pierced later. The "guaranteed cash flow" ' $t \mapsto W^{\odot}(t)$ is the one associating with each date the minimum guaranteed investment. The "guaranteed cushion $W^{\odot}(t)-\mathbf{k}(t) \geq 0$ is the difference between the minimum guaranteed investment and the floor.

The initial guaranteed cushion provides a new measure of risk summarized in a single number. This number has an explicit meaning and is immediately usable : insurance premium, net present value [NPV], economic capital, which, in [41, Crouhy, Galai \& Mark], « measures [...] risk $\gg$, p.15, « is the financial cushion [...] to absorb unexpected losses $\gg$, p. 258, « capital is also used to absorb risk $\gg$, p. 366 , etc.

There are parallels and differences between stochastic and tychastic approaches. Regarding viability and capturability issues, the stochastic case is a (too) special particular case of the tychastic one viability (See Section 7, p. 34).

This study deals only with tychastic uncertainty, since stochastic uncertainty has been extensively developed over a century.

\subsection{Contingent Uncertainty}

The question arises whether tychastic uncertainty "offsets" contingent uncertainty.

In fact, the minimum guaranteed investment increases when the "tychastic reservoir" $\mathcal{R}(t)$ increases, that is to say, in this study, when the lower bound of the underlying return decreases. Is it possible to act on exposure $E(t)$ by choosing to invest more or less underlying shares?

The tychastic reservoir $t \leadsto \mathcal{R}(t)$ of tyches (underlying returns) can be contrasted with the "contingent reservoir" $\widetilde{\mathcal{E}}$ of "management rules" $(t, W) \mapsto \widetilde{E}(t, W)$ available to investors (or mechanisms of regulation), to draw from contingent reservoir.

The word contingent comes from the Latin verb contingere, to arrive unexpectedly or accidentally. Aristotle meant by automaton, a concept dating back at least to Epicurious, one of the first rebel against the determinism of his predecessors.

Chen Luxi the offers Chinese translation "self, born", 自发, where 自, means

«by himself $»$, whereas 发, means "produce" or "born. Today, these automata became 
the feedbacks to be selected in the contingent reservoir.

We adopt here the definition of contingency given by Leibniz : "Contingency is a nonnecessity, a characteristic attribute of freedom."

In this study, contingent uncertainty is described by financial constraints on exposures, which require that each period $t$

$$
\forall t \in[0, T], \forall W \geq 0, \quad E \in\left[E^{b}(t, W), E^{\sharp}(t, W)\right]
$$

$$
\begin{aligned}
& \text { Example - If } \\
& \qquad\left\{\begin{array}{cl}
B(t) \geq 0 & \text { is the target allocation } \\
A(t) \geq B(t)-1 & \text { is the maximum cash }-P^{0}(t) S^{0}(t)
\end{array}\right.
\end{aligned}
$$

we set

$$
E^{b}(t, W):=B(t) W(t) \text { and } E^{\sharp}(t, W):=(1+A(t)) W(t)
$$

If $A(t)=-|A(t)|<0$, condition $-P^{0}(t) S^{0}(t) \leq A(t) W$ can be written $P^{0}(t) S^{0}(t) \geq$ $|A(t)| W$, which means that the portfolio should include a minimum share of the monetary value of the portfolio.

These bounds $A(t)$ and $B(t)$ describe more or less severe prudential constraints.

Viability theory allows us to calculate

1. the VPPI management rule $(t, W) \mapsto E^{\mathcal{Q}}(t, W)$ (Viabilist Portfolio Performance and Insurance) which depends on tychastic uncertainty represented by the function $t \mapsto$ $R^{b}(t)$ in the predictive approach;

2. the FPPI management rule $(t, W) \mapsto E^{\diamond}(t, W)$ (Viabilist Impulsive Portfolio Performance and Insurance) that depends on loans authorized in the impulse approach.

They provide in each case the exposure of the portfolio such that the value $W(t)$ of the portfolio is always greater than the floor. These two approaches are then the inverse to each other.

\section{Example : CPPI (Constant Proportional Portfolio Insurance)}

This CPPI management rule is defined by

$$
\widetilde{E}(t, W):=m(W-\mathbf{k}(t)) \text { where } m>0 \text { is called the "multiplier" }
$$

Attractive for its simplicity and the fact that the choice of the management rule amounts to choosing the multiplier $m$, we know that this rule does not guarantee anything, since the floor may be pierced in some cases. It is the same of its many variants which introduced time dependent multiplier $m(t)$ or even more sophisticated functions, for the simple reason that 
their choice is not dictated by the problem : eliminate the possibility of piercing the floor. The initial cushion (which we propose for measuring the risk in this problem) is not calculated in practice for the CPPI, but just estimated by statistical methods. For this reason, it happens that the value of the portfolio pierces the floor for some realizations of the underlying prices.

The direct approach means that the choice of a feedback is made a priori or ex ante is made at initial investment date. This approach is, by far, the most often used in science : in economics, it is the law of supply and demand (Walrasian tâtonnement); in Artificial Intelligence, it is the Hebb law describing a a priori learning mechanism regardless what must be learned; in robotics, it is usually a linear "feedback", which does not necessarily take into account either the non-linearity of mechanical systems or the constraints, etc..

The construction of the VPPI management rule is an example of a inverse approach : management rule is a solution to the problem instead of being a datum.

Unfortunately, the VPPI management rule $(t, W) \mapsto E^{\odot}(t, W)$ does not enjoy the simplicity of the CPPI management rule (defined by a simple closed linear analytical formula), but is tabulated by the VPPI software that computes the values of the portfolio and its exposure to each date. However, we can deduce an ad hoc multiplier. It is not explicit, but concealed in the dark and invisible computer memory.

The mathematical tradition of the era that preceded the advent of computers in the middle of last century required mathematical results to be expressed in explicit analytical mathematical formulas needed to calculate it numerically "by hand" through the various tables of "special functions". A treat for the mathematicians, but very often at exorbitant price of much too restrictive assumptions. This tradition of "the search for the lost formula" is no longer justified since it is possible to develop suitable algorithms and software for obtaining numerical information in the absence of explicit formulas. This what does matter.

The investor must :

- have confidence in the conclusions of mathematical theorems which he can not always prove by himself;

- validate the relevance of the conclusion to the problem of interest,

- and, above all, appreciate the "cost" and the relevance of assumptions in terms of the "value" of the conclusion.

In this case, it may be reassured because he is really insured; the "I" of VPPI is perfectly legal. 


\section{Predictive and Impulse Approaches}

These two approaches are inverse.

1. predictive approach : it uses a prediction model of the lower bounds of underlying returns as an input (tychastic risk assessment) from which are computed

(a) the contingent management rule providing at each date the exposures $E^{\mathcal{Q}}(t, W)$;

(b) minimum guaranteed (IGM) $W^{\odot}(t)$ at each date (insurance)

for obtaining values of the portfolio above the floor.

2. impulse approach : it uses as input a cash flow of authorized from which are computed

(a) the impulse management rule providing the exposures $E^{\diamond}(t, W)$;

(b) guaranteed minimum returns (GMR) $R^{\diamond}(t)$

for driving values of the portfolio above the floor.

\subsection{Predictive Approach}

It requires the knowledge at investment date of the tychastic map described in this study by the function $t \mapsto R^{b}(t)$ of "lower bounds of underlying returns".

The investor is supposed to provide the lower bounds $R^{b}(t)$ of the returns obtained, for example, by prediction or extrapolation method.

However, VIMADES proposes the VIMADES Extrapolator designed from two properties of Schwartz distributions :

2 VIMADES Extrapolator. It is a mathematical technique that allows to extend historical evolution defined on an interval $[-H, 0]$ called extrapolator depth. The originality of the VIMADES Extrapolator is to take into account not only past values of the historical evolution, but also those of its derivatives of order less than or equal to a given order. They thus describe the trends (velocity, acceleration, jerk, etc.) These past trends are taken into account by the VIMADES Extrapolator.

Extrapolating each historical (past) time series of upper bounds $(H I G H)$ and lower bounds $(L O W)$ of the underlying prices provided daily by brokerage firms, the VIMADES Extrapolator calculates lower bounds of underlying returns of the future needed for the computation of the VPPI management rule. No need of a "volatilometer" for measuring volatile volatilities.

Example : In all examples, we chose the period from September 3 (investment date) to December 10, 2008 (exercise date), the most volatile period of the "subprime crisis". 


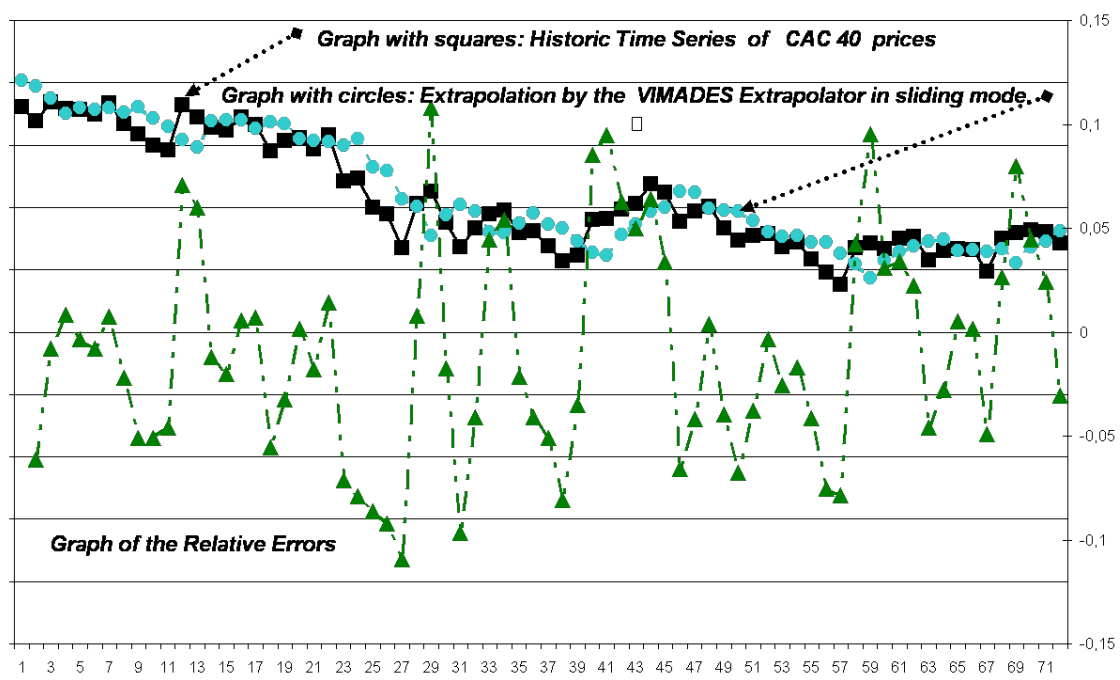

3 Example of extrapolation by the VIMADES Extrapolator. The historical series is the CAC 40. The extrapolation is obtained by "sliding" at each date the VIMADES Extrapolator on previously recorded data. The graph of the relative errors is displayed on another scale. It increases during the most volatile period of the subprime crisis.

The value of the portfolio is governed by a (very simple) tychastic controlled system, where controls are the exposures $E(t, W) \in\left[E^{b}(t, W), E^{\sharp}(t, W)\right]$ of the portfolio and the tyches are the returns $R(t) \geq R^{b}(t)$ of the underlying :

$$
\begin{cases} & \forall t \in[0, T] \\ (i) & W^{\prime}(t)=R^{0}(t) W(t)+E(t)\left(R(t)-R^{0}(t)\right) \text { (evolutionary engine) } \\ (\text { ii }) & E(t) \in\left[E^{b}(t, W(t)), E^{\sharp}(t, W(t))\right] \text { (controls) } \\ (\text { iii }) & R(t) \geq R^{\sharp}(t) \text { (tyches) }\end{cases}
$$

subject to the floor constraints

$$
\forall t \in[0, T], W(t) \geq \mathbf{k}(t) \text { and } W(T)=\mathbf{k}(T)
$$

Definition 3.1 Minimum Guaranteed Investments. Suppose that the floor $t \mapsto \mathbf{k}(t)$ and the bounds $\left[E^{b}(t, W), E^{\sharp}(t, W)\right]$ describing the contingent uncertainty are given. Assume known the lower bounds $R^{b}(t)$ of the returns on the underlying describing tychastic uncertainty. The problem is to find each date $t$

1. the (exposure) management rule $E^{\complement}(t, W) \in\left[E^{b}(t, W), E^{\sharp}(t, W)\right]$; 
2. the minimum guaranteed investment $(M G I) W^{\mathcal{Q}}(t)$;

3. and in particular the initial minimum guaranteed investment ("viability insurance") $W^{\odot}(0)$

such that

1. starting at investment date 0 from $W_{0} \geq W^{\ominus}(0)$, then regardless be the evolution of tyches $R(t) \geq R^{b}(t)$, the value $W(t)$ of the portfolio is governed by the management module

$$
W^{\prime}(t)=R^{0}(t) W(t)+E^{\ominus}(t, W(t))\left(R(t)-R^{0}(t)\right) \text { (VPPI management module) }
$$

is always above the floor, and, actually, to the minimum guaranteed investment ;

2. starting at investment date 0 from $W_{0}<W^{\ominus}(0)$, regardless the management rule $\widehat{E}(t, W) \in\left[E^{b}(t, W), E^{\sharp}(t, W)\right]$ (including the CPPI management rule and its variants), there exists at least one evolution of returns $R(t) \geq R^{b}(t)$ for which the value of the portfolio managed by

$$
W^{\prime}(t)=R^{0}(t) W(t)+\widehat{E}(t, W(t))\left(R(t)-R^{0}(t)\right)
$$

pierces the floor.

Remark : Management Module with Ratchet. The ratchet is a mechanism that prohibits a process to go backward once a certain threshold is exceeded to force it to move forward. In finance, a ratchet mechanism allows management to irreversibly reap profits for a certain period.

For describing mathematically this mechanism, consider two parameter $\nu \geq 0$ et $\alpha \in[0,1]$ with which we associate the ratchet operator mapping investments $W$ and $W^{\odot}$ to the "ratchet value" defined by

$$
\mathbb{C}\left(\nu, \alpha ; W, W^{\odot}\right):=\left\{\begin{array}{cc}
(1-\alpha) W+\alpha W^{\odot} & \text { if } \\
\max \left(W, W^{\odot}\right) & \text { otherwise }
\end{array} \quad W-W^{\odot} \geq \nu\right.
$$

Consequently

$$
\mathbb{C}\left(\nu, \alpha ; W, W^{\curvearrowright}\right) \in\left[W, W^{\curvearrowright}\right]
$$

For example, if $\alpha=0$, then $\mathbb{C}\left(\nu, 0 ; W, W^{\odot}\right)=\max \left(W, W^{\odot}\right)$ and if $\alpha=1$, $\mathbb{C}\left(\nu, 1 ; W, W^{\odot}\right)=W^{\odot}$ if $W-W^{\odot} \geq \nu$.

The "benefit" defined by $W-\mathbb{C}\left(\nu, \alpha ; W, W^{\odot}\right)$ is equal to $(1-\alpha)\left(W-W^{\odot}\right)$ if $W-W^{\odot} \geq \nu$ and is equal to 0 if $0 \leq W-W^{\odot}<\nu$. 
4 Guaranteed Ratchet Management Module. The values $W(t)$ of the portfolio governed by the guaranteed ratchet management module defined by

$$
\left\{\begin{array}{l}
W^{\prime}(t)=R^{0}(t) \mathbb{C}\left(\nu(t), \alpha(t) ; W(t), W^{\odot}(t)\right)+E^{\odot}(t, W(t))\left(R(t)-R^{0}(t)\right) \\
\text { (guaranteed ratchet management module) }
\end{array}\right.
$$

are always above the floor whenever, for all $t \in[0, T], R(t) \geq R^{b}(t)$.

Example : The MGI of the CPPI management rule. Statistical methods do not provide a guaranteed initial cushion (portfolio insurance (PI)) for the portfolio governed by the stochastic CPPI management rule. However, in the tychastic case, we can always associate with the floor and the CPPI tychastic system

$$
W^{\prime}(t)=W(t) R^{0}(t)+m(t)(W(t)-\mathbf{k}(t))\left(R(t)-R^{0}(t)\right)
$$

(which is no longer controlled by exposures) the initial CPPI guaranteed investment above which, whatever the returns above the lower bounds, the value of the portfolio governed by the tychastic system is above the floor at each date. Actually, we should use the slightly modidied CPPI modified rule $\bar{E}(t, W):=\max (0, W-\mathbf{k}(t))$ for using differential equation $W^{\prime}(t)=W(t) R^{0}(t)$ whenever the value of the portfolio hits the floor :

$$
\left.W^{\prime}(t)=W(t) R^{0}(t)+m(t) \max (0, W(t)-\mathbf{k}(t))(t)\right)\left(R(t)-R^{0}(t)\right)
$$

Naturally, since the VPPI management rule $(t, W) \mapsto E^{\mathcal{O}}(t, W)$ provides the minimum guaranteed investment, the CPPI one is always larger than or equal to $W^{\curvearrowright}(0)$ (see Figure (11), p. 23 for an illustration of this property).

\subsection{Impulse Approach}

We have assumed up to now that it existed some lower limits to the underlying returns (the worst case) when the lower bounds $R^{b}(t)$ are known. It is from that knowledge that it has been possible to determine the VPPI management rule $E^{\complement}(t, W)$ and the minimum guaranteed investment $W^{\curvearrowright}(t)(t)$.

Beginning with an investment higher than or equal to the minimum guaranteed investment, it may happen that, for underlying returns smaller than those that were forecasted, the value of the portfolio pierces the floor. 
Since it may be difficult to determine the lower bounds $R^{b}(t)$, the question arises to address the inverse problem. Instead of computing the minimum guaranteed investment $W^{\curvearrowright}(t)$, we assume instead known authorized minimum investment $\mathbf{h}^{\diamond}(t) \geq \mathbf{k}(t)$ and we derive upper bounds $R^{\diamond}(t)$ of underlying returns guaranteeing that the floor will never be pierced.

This possible by using an impulse management rule allowing the investor to set instantly by an impulse (infinite velocity) a new higher level of investment by using its right to borrow, and start again from the minimum guaranteed investment.

Therefore the impulse tychastic system operates as a usual regulated tychastic system

$$
W^{\prime}(t)=R^{0}(t) W(t)+E^{\diamond}(t, W(t))\left(R(t)-R^{0}(t)\right)
$$

but, requiring further that at each time $t$ when the value $W(t)=\mathbf{k}(t)$ hits the floor, then it is reset to $W(t)=\mathbf{k}^{\diamond}(t)$. See [35, Bensoussan \& Lions] [36, Bensoussan \& Menaldi], [5, Aubin], [14, 16, 15, Aubin \& Haddad], [17, Aubin, Lygeros, Quincampoix, Sastry \& Seube], etc.

Naturally, this impulse management mode is not viable when the returns are too low. It is therefore needed to compute the Guaranteed Minimum Returns according to authorized loans instead of forecasting lower bounds on underlying returns.

In other words, we no longer attempt to predict the disaster (transgression of the constraint, here, piercing the floor), but rather to build a reset feedback to remedy the constraint violations. The techniques of impulse viability allow us to derive and compute tychastic uncertainties needed to make viable the impulse management module.

The FPPI (Viabilist Impulse Portfolio Performance and Insurance) management approach is exactly the inverse of the predictive management :

1. First, we assume known the authorized minimum investment $\mathbf{h} \diamond(t) \geq \mathbf{k}(t)$ at each date $t$ (or authorized loans $\pi^{\diamond}(t):=\mathbf{h}^{\diamond}(t)-\mathbf{k}(t) \geq 0$ ) and we are looking for

(a) a (exposure) management rule $E^{\diamond}(t, W) \in\left[E^{b}(t, W), E^{\sharp}(t, W)\right]$ of exposure;

(b) the guaranteed minimum return (GMR) $R^{\diamond}(t)$, lower bounds of returns of the underlying above which the value of the portfolio remains higher than the floor.

2. impulse management $:$ if at date $t$, the value $W(t):=\mathbf{k}(t)$ reaches the floor, the investor borrows $\pi^{\diamond}(t)$ and switches immediately to the value $W(t):=\mathbf{h}^{\diamond}(t)$ of the portfolio.

As a result, instead of forecasting lower bounds $R^{b}(t)$ of future underlying returns, either by statistical methods, or by using the VIMADES extrapolator of lower and upper bounds of past prices of the underlying, impulse management assumes known in advance the authorized investments (or loans) and compute the Guaranteed Minimum Returns $R^{\diamond}(t)$. 
Definition 3.2 Guaranteed Minimum Returns. Suppose that the floor $t \mapsto \mathbf{t}(t)$ and the bounds $\left[E^{b}(t, W), E^{\sharp}(t, W)\right]$ describing the contingent uncertainty are given. Consider the amount $\mathbf{h}^{\diamond}(t) \geq \mathbf{k}(t)$ of authorized investments (or authorized loans $\pi^{\diamond}(t):=\mathbf{h}^{\diamond}(t)-$ $\mathbf{k}(t) \geq 0$ for borrowing $\left.\pi(t):=\mathbf{h}^{\diamond}(t)-W(t) \leq \pi^{\diamond}(t)\right)$.

The goal is to find each date $t$

1. the management rule of exposures $E^{\diamond}(t, W) \in\left[E^{b}(t, W), E^{\sharp}(t, W)\right]$;

2. Guaranteed Minimum Return $R^{\diamond}(t)$

such that, starting from $W_{0} \geq \mathbf{k}(0)$ and knowing the return $R(t) \geq R^{\diamond}(t)$ of the underlying, the impulse management module

$$
W^{\prime}(t):=\left\{\begin{array}{lll}
R^{0}(t) W(t)+E^{\diamond}(t, W(t))\left(R(t)-R^{0}(t)\right) & \text { if } \quad W(t) \geq \mathbf{h}^{\diamond}(t) \\
R^{0}(t) \mathbf{h}^{\diamond}(t)+E^{\diamond}(t, W(t))\left(R(t)-R^{0}(t)\right) & \text { if } \quad \mathbf{k}(t) \leq W(t) \leq \mathbf{h}^{\diamond}(t)
\end{array}\right.
$$

manages a portfolio the value of which is always above the floor.

Remark : Tychastic Reliability — The approach provides an answer to a problem that could be called "tychastic reliability" as it provides lower bounds of returns (describing the boundary of the tychastic map) above which the guarantee sought (the value of the portfolio must be greater than the floor) and the means of ensuring it (by paying for a cash flow higher than the floor) to be reliable reliable at $100 \%$. This question opens a new chapter in viability theory (see Section 6.3 , p. 30 for some details).

Remark : Tychastic reliability and probability of ruin - It is possible to interpret otherwise the impulse management mode, regarding $\mathbf{h}^{\diamond}(t)$ as the liability and $\mathbf{k}(t) \leq \mathbf{h}^{\diamond}(t)$ as a tolerance to ruin. Instead of trying to compute the probability of ruin tolerance, we seek and obtain the Guaranteed Minimum Return which forbids to go beyond that tolerance to ruin. The framework of "Solvency 2", for example, requires that the difference between the value of portfolio assets and provisions to hedge liabilities must be positive at every date, possibly with a "probability of failure" (where equity is negative) below a given threshold. In our framework, the probability of ruin is replaced by the Guaranteed Minimum Returns (GMR). 


\section{Examples}

In all the examples that follow, we chose the period from 3 September (investment date) to December 10, 2008 (exercise date) of the "subprime crisis", as underlying the CAC 40 ( see Figure 3, p. 14), and as riskless asset the Euro Over Night Index Average (EONIA). The chosen floor is "variable annuities" floor described in Figure??, p. ?? . This example can regarded as a stress test, at least for the Extrapolator of VIMADES which did not always predict the volatile changes of the actual returns of the underlying during this crisis. Since the assumption is wrong, so were the conclusions : for four dates, the value of the portfolio went below the Minimum Guaranteed Investment, but remained above the floor. However, we had to use the impulse management module at those dates when the portfolio was no longer self-financed.

\subsection{Predictive Case}

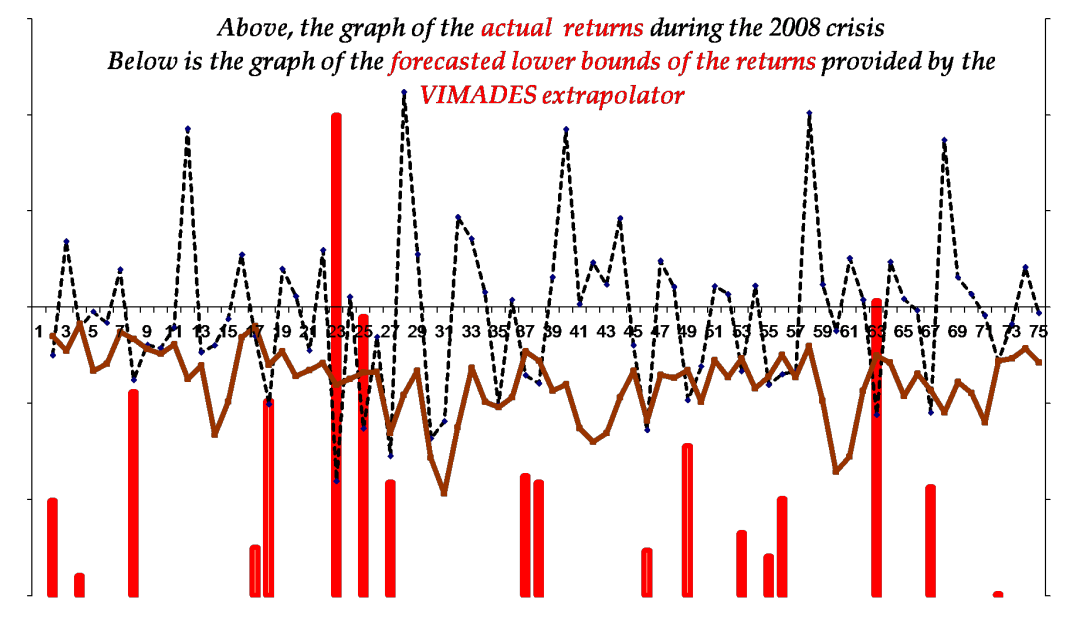

The histogram provides the date when the forecast was wrong and the amount of the error

5 Realized returns and lower bounds of returns obtained with the VIMADES extrapolator. The bottom curves shows the calculated lower bound $R^{b}(t)$ of the returns obtained from extrapolations of depth four higher and lower of the CAC 40 values with the VIMADES extrapolator (taking into account the price, velocity, acceleration and jerk). The top curves shows the returns $R(t)$ of the CAC 40 observed ex-post. During this period which was the most volatile of the subprime crisis, there have been observed returns below the lower bound returns calculated by the extrapolator. Dates and values of the prediction error are indicated by the histogram.

The robot-trader VPPI calculates at each date the minimum guaranteed investment (MGI) which ensure that the floor will never be pierced. 


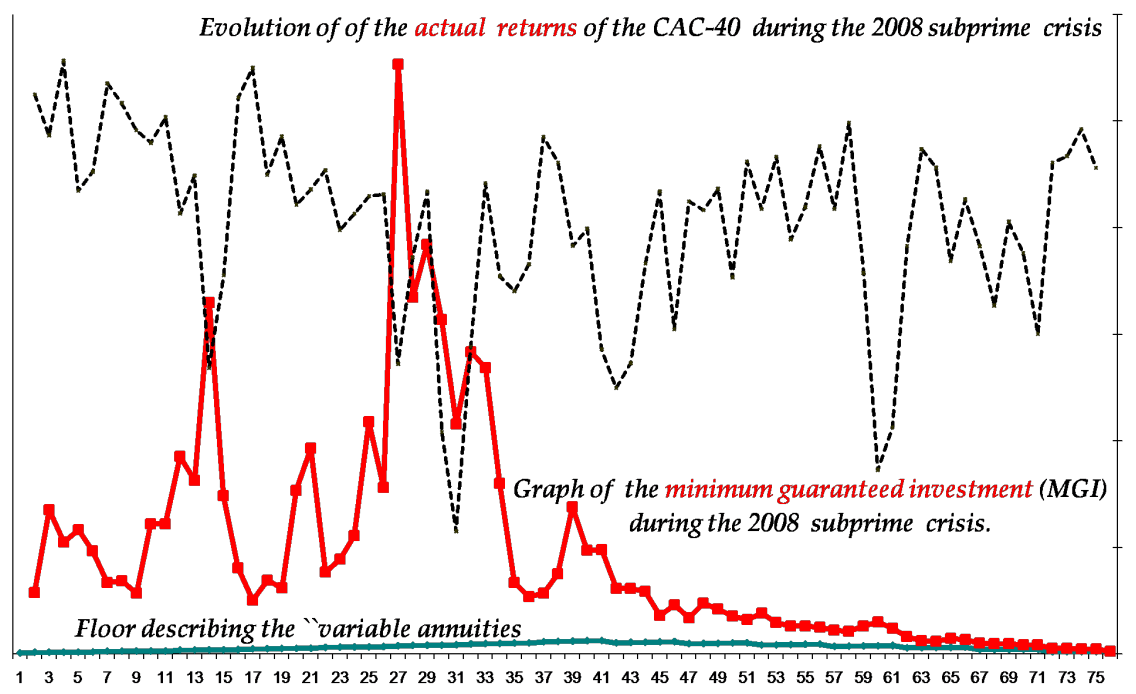

6 Floor and MGI. The bottom curve represents the floor $\mathbf{k}(t)$ that should never be pierced by the portfolio value. The top curve $W^{\ominus}(t)$ represents the minimum guaranteed investment, which depends only on the floor, the riskless return and lower bounds of underlying returns obtained by the VIMADES extrapolator (see Figure 5).

The VPPI management module calculates "opportunistically" the values of the portfolio depending on the effective realizations of the underlying prices. This is the case when these returns are higher than the lower bounds of performance.

As seen in Figure 5, this has not always been the case during the subprime crisis. However, it is interesting to test this example, even if we know that it is possible that the portfolio dives under minimum guaranteed investment and then breaks through the floor for at least one evolution of underlying returns. This is not due to a failure of the VPPI management rule, since the assumption $R(t) \geq R^{b}(t)$ is not satisfied for some dates $t$.

However, we can use the impulse management module using as authorized investment $\mathbf{k}^{\diamond}(t):=W^{\ominus}(t)$ in the FPPI software. In this case, the floor will not be pierced, but this will require to borrow loans at four dates. 


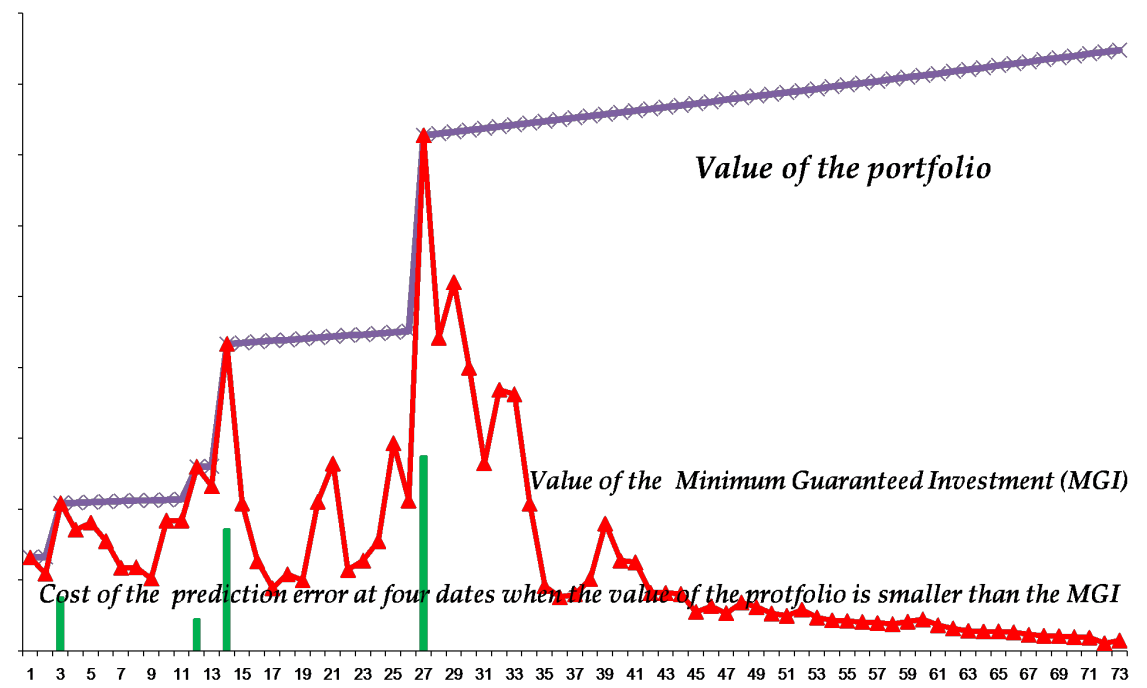

7 Impulse Management. The bottom curve is authorized investment chosen to be the minimum guaranteed investment computed by the VPPI using the lower bounds obtained by the VIMADES extrapolator which provided wrong forecasts. We set $\mathbf{k}^{\diamond}(t):=W^{\ominus}(t)$ and use the impulse management module to calculate the additional loans whenever the value of the portfolio becomes smaller than the minimum guaranteed investment (MGI).

The management module determines each day the portfolio's exposure, from which we calculates the number of shares of the underlying :

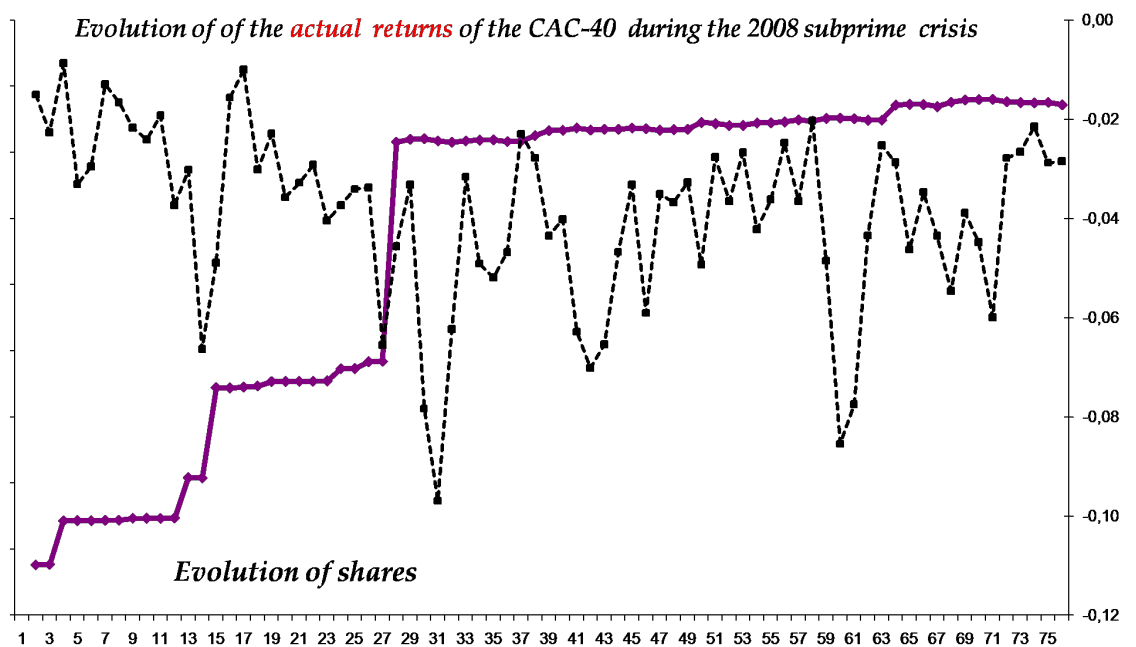

8 Shares of the Underlying. Once the VPPI management rule is calculated and stored in the computer memory, the management module provides the number of shares of the 
underlying within its portfolio based on the realization of prices. The figure represents the evolution of the number of shares.

The VPPI provides also the values obtained under ratchet :

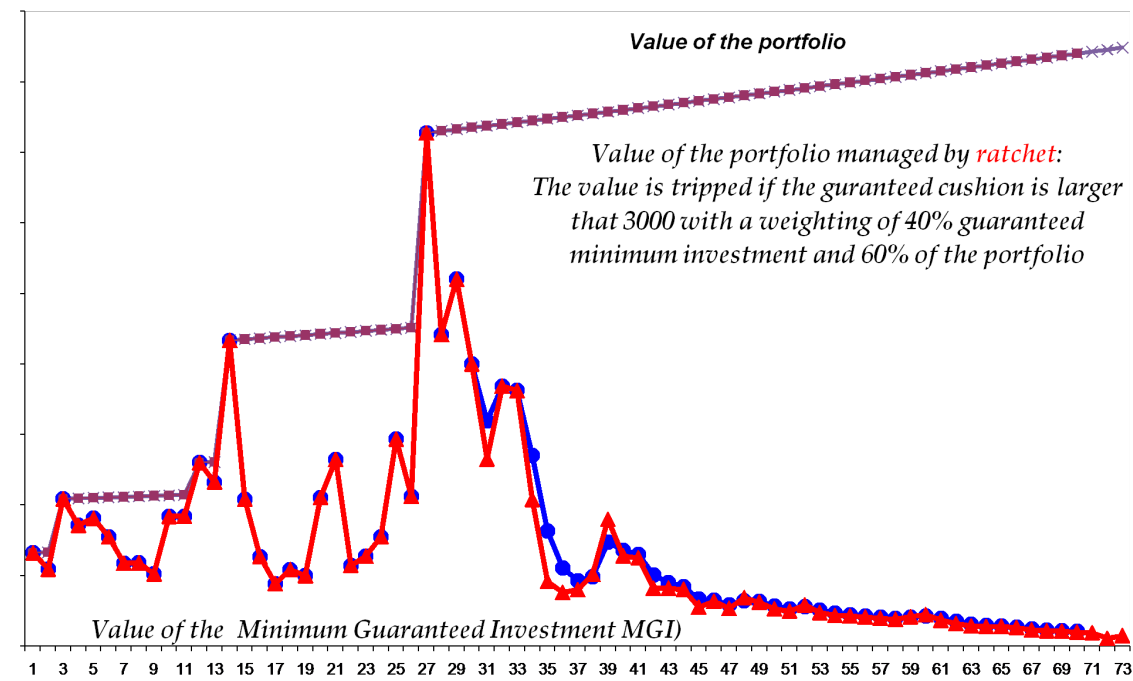

9 Ratchet Management. Example of management triggered whenever the guaranteed profit (difference between the value of the portfolio and the minimum guaranteed investment) is greater than a given threshold to sell irreversibly (ratcheting) a fixed percentage of that profit.

We now illustrate the influence of the prediction mechanism on the MGI :

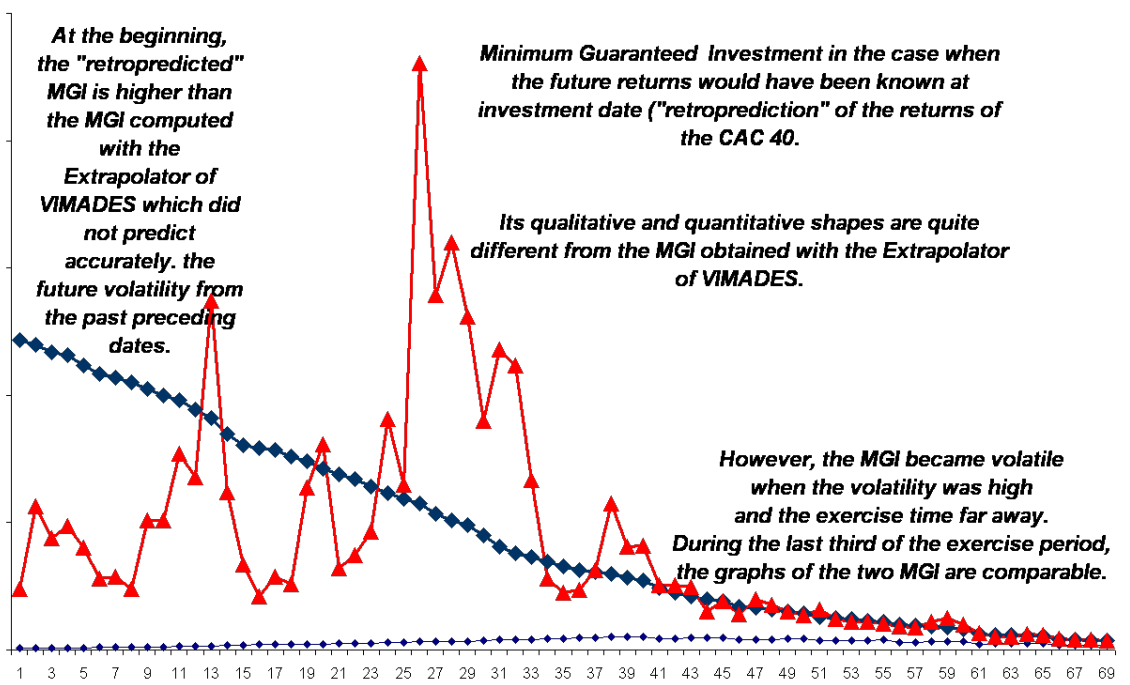


10 Comparison of the MGI's between the predicted and "retropredicted" lower bounds of returns. We recall that, essentially, the value of Minimum Guaranteed Investment depends on the floor and the (sliding) predictions of the future lower bounds of the return of the underlying up to exercise time. It is then interesting to compute the MGI ex-post, i.e., when the future is "retropredicted" at investment date.

Before ending this study, we shall compare on this example the minimum guaranteed investment when the dynamics governing the value of the portfolio in the tychastic choice. We can prove that the VPPI management rule is the one yielding at each date the minimum guaranteed investment. Yet, the same approach can compute the minimum guaranteed investment for each the dynamics using a fixed decision rule, such as the CPPI :

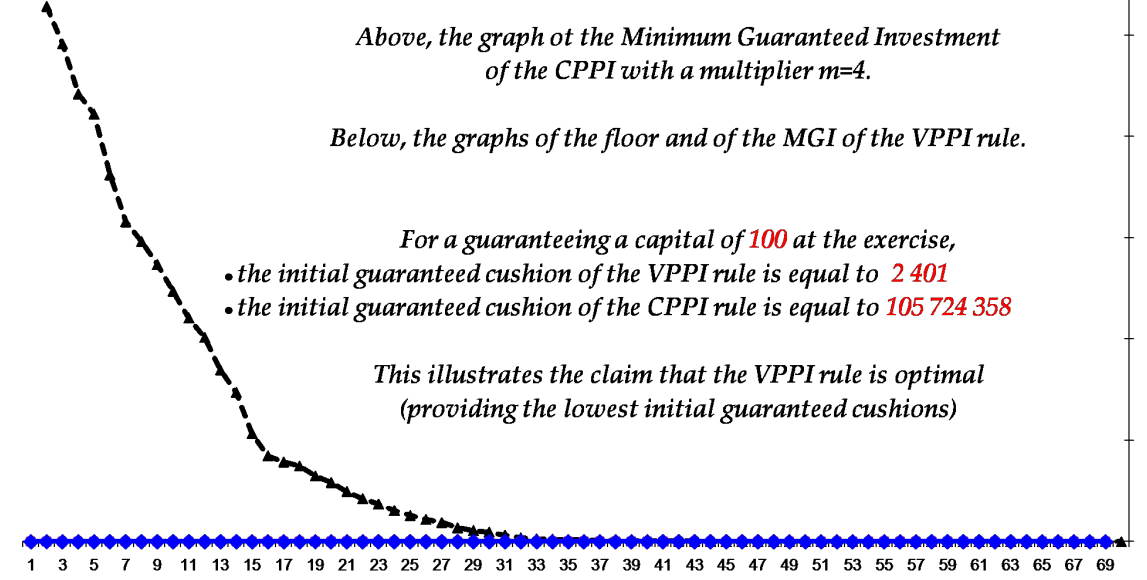

11 Comparison between the MGI of the CPPI and VPPI management rule. We have proved that the VPPI management rule is the one providing the minimum investment guaranteeing that the value of the portfolio is always larger that the floor whatever returns above the lower bounds of the underlying returns. However, we can associate with any management rule its guaranteed initial cushion. This is what was done, under the same conditions, by taking the CPPI rule with a multiplier equal to 4. During the second half of the exercise period, the MGI of the CPPI rule was always above the MGI of the VPPI rule, an illustration of the theorem. However, it was not able to provide reasonable insurance at the beginning of the volatile period.

\subsection{Impulse Case}

We present an example of the impulse approach. 


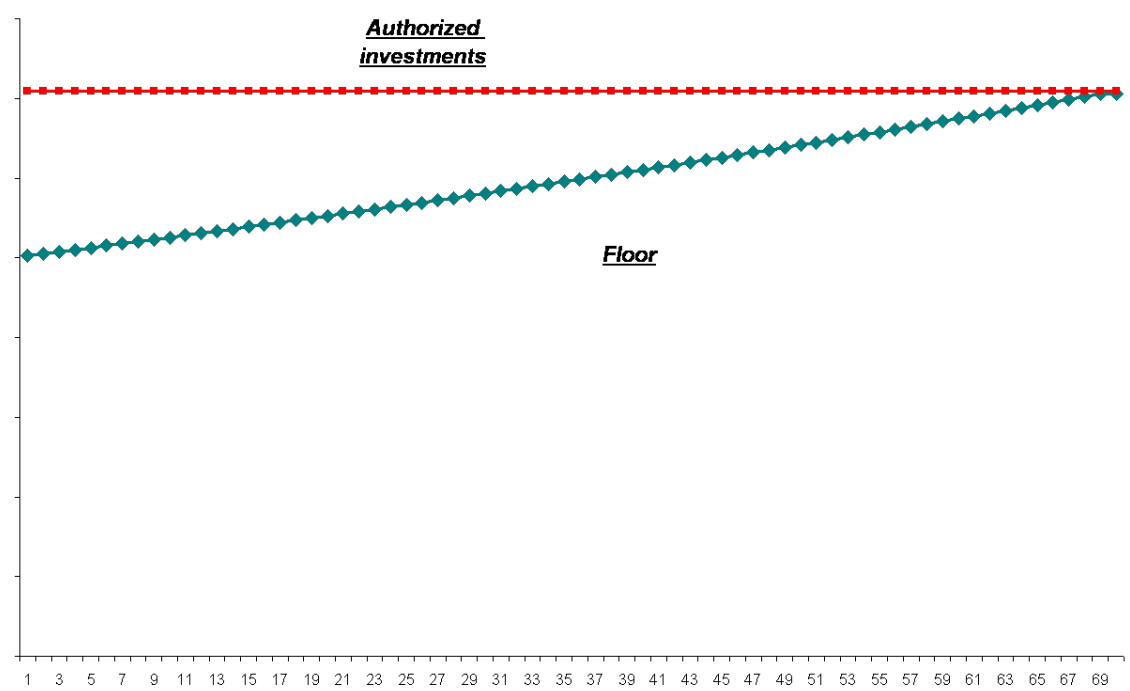

\section{Example of authorized investments.}

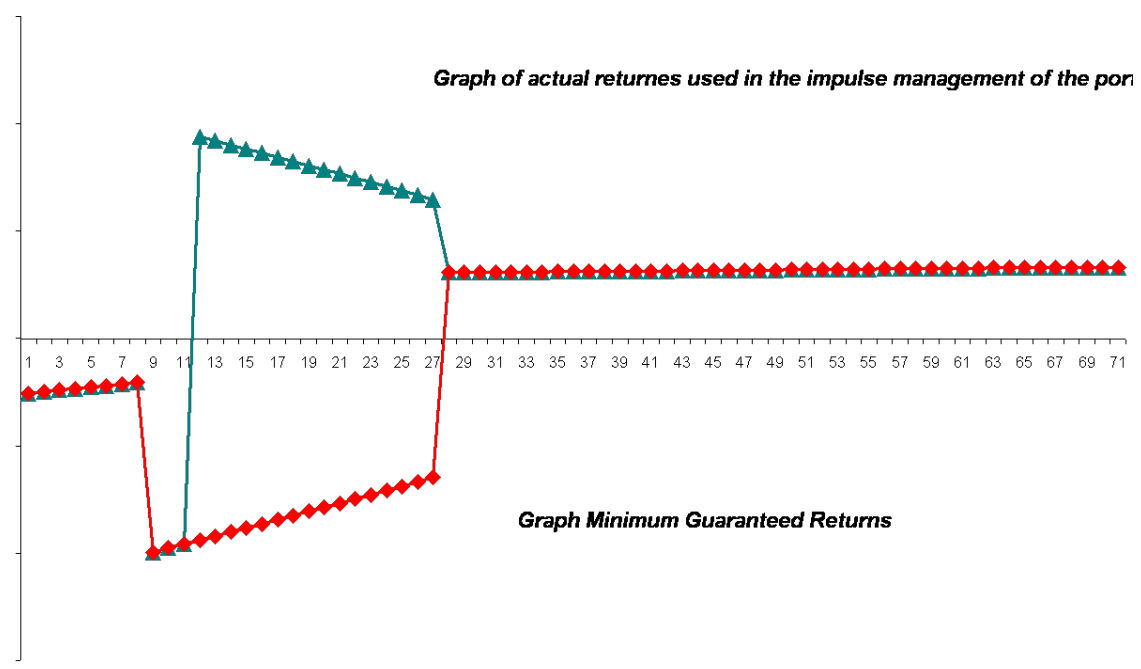

13 Guaranteed Minimum Returns. This figure displays the Guaranteed Minimum Returns derived from the knowledge of the floor, the authorized investments and the return of the riskless asset (lower curve), It also shows an example of actual returns above the Guaranteed Minimum Returns. 


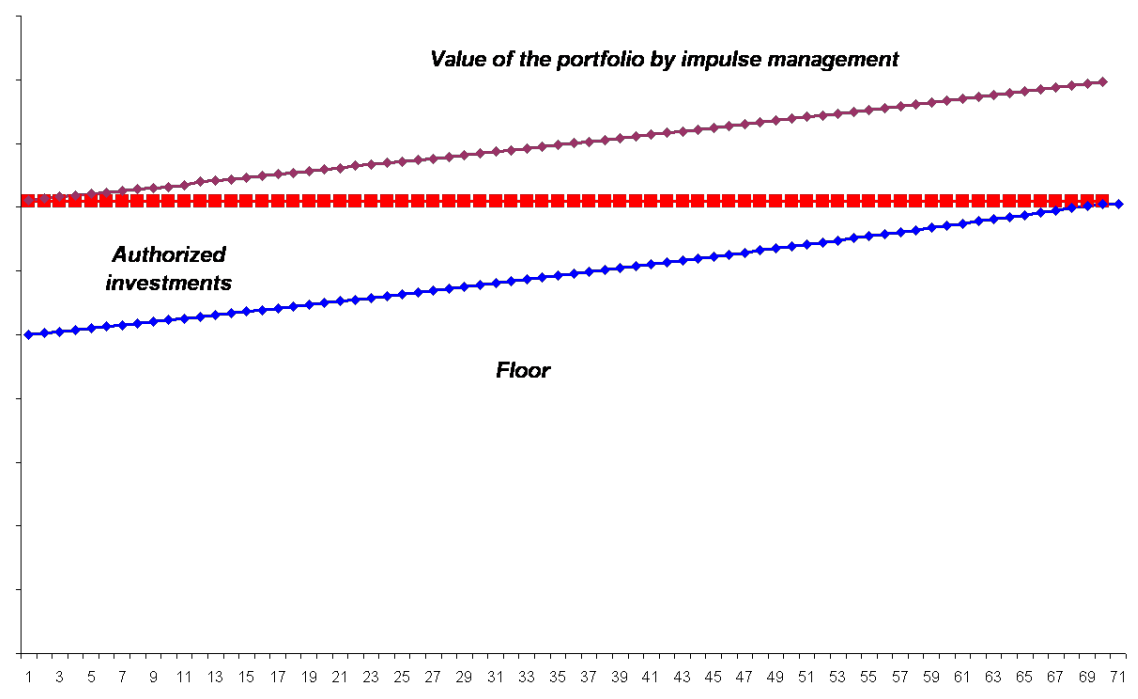

14 Impulse Management. This figure represents the impulse management of the portfolio obtained from actual returns shown in Figure.

\section{The VPPI and FPPI management software}

One way to summarize the VPPI structure is to provide the flow charts of the viability software to solve this problem. They show the division of the programme into two steps :

1. the computation of the Minimum Guaranteed Investments and Guaranteed Minimum Returns and their management rules;

2. the use of the management rules for managing the value of the portfolio knowing at each date the actual underlying return.

The first step is the discretization of continuous time by discrete dates, functions by sequences and reformulate the data and concepts in this discrete framework (this step is not needed if the problem is directly formulated in discrete time, as it is often the case).

Then algorithms are used to calculate iteratively guaranteed capture basin of targets viable in an environment and the feedback rule. It uses techniques of set-valued numerical analysis handling discrete subsets (grids) mostly based the lattice properties of guaranteed capture basins.

These algorithms are used in the VPPI and FPPI software. We describe below their flow charts. They indicate what are the inputs and outputs of the software, presented in the form of .csv files. 


\subsection{Flow Chart of the VPPI Software}

\section{Minimum Guaranteed Investment}

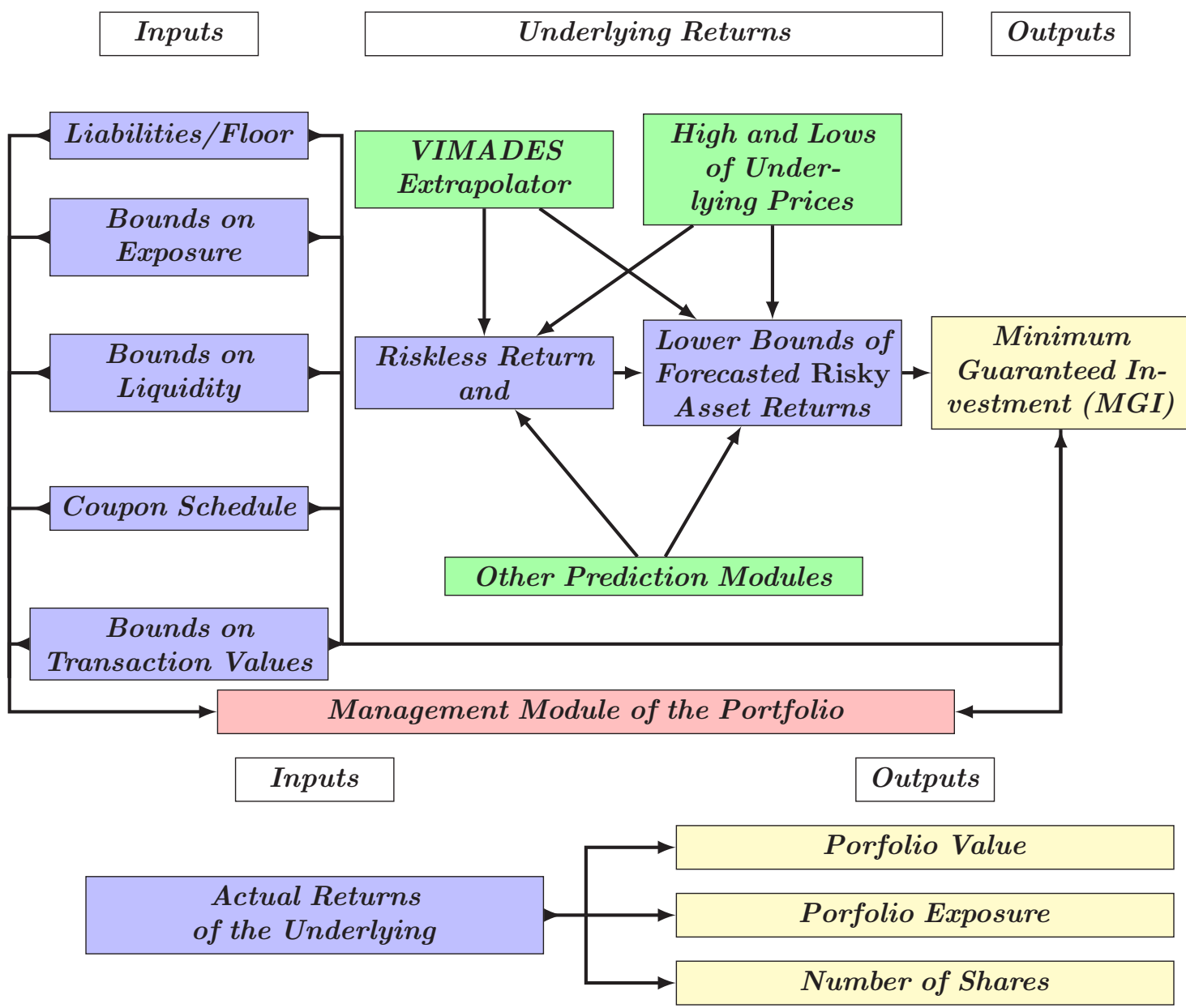




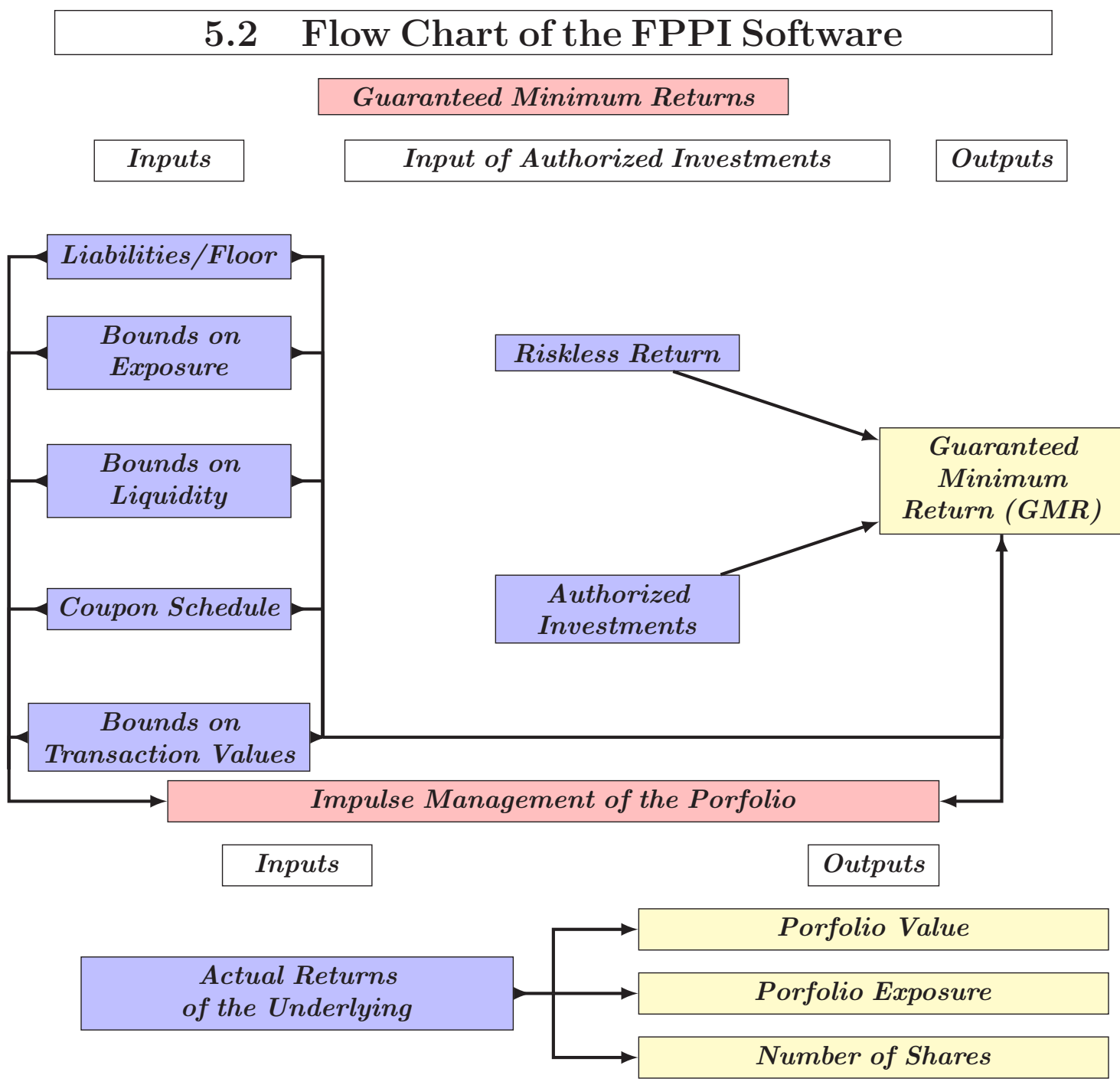

\section{Guaranteed Viability Kernels}

We present very briefly the mathematical concepts of viability theory used to solve the cushion guaranteed ALM management. For defining controlled tychastic systems, we need to make precise the concept of feedback :

15 Retroactions and loops. Consider a set-valued map $x \leadsto R(x)$ (interpreted as a contingent set-valued map).

1. A retroaction $(t, x) \mapsto \widetilde{r}(t, x) \in R(x)$ is a single-valued selection of contingent set-valued 
map.

2. A retroaction $t \mapsto \widetilde{r}(t) \in R(x(t))$ is said to "open loop" if it does not depend on the state.

3. A retroaction $x \mapsto \widetilde{r}(x) \in R(x)$ is termed feedback or "closed loop" if it depends only the state

By abuse of language, we say simply feedback that $(t, x) \mapsto \widetilde{r}(t, x) \in R(x)$ governs the evolution of a system $x^{\prime}(t)=f(x(t), u(t))$ where $u(t) \in R(x(t))$ if this evolution is governed by the ordinary differential equation

$$
x^{\prime}(t)=f(x(t), \widetilde{r}(t, x(t)))
$$

We denote by $\widetilde{R}$ a set of retroactions.

Regarding open loop' feedbacks, the largest class $\widetilde{R}$ is the class of "measurable selections" of the set-valued map $R$ (see [13, Aubin \& Frankowska] for selection and parametrization theorems). It is this class of feedbacks that is implicitly used when one speaks of differential inclusion

$$
x^{\prime}(t) \in f(x(t), R(x(t))):=\bigcup_{r \in R(x(t))} f(x(t), r)
$$

Michael's theorem provides sufficient conditions for obtaining continuous selections $x \mapsto$ $\widetilde{r}(x) \in R(x)$. The search of selection and parameterizations of set-valued maps is a substantial chapter of mathematics.

\subsection{Tychastic Systems}

\section{Consider}

1. a vector space $X:=\mathbb{R}^{d}$ (interpreted as a state space) and a vector space $\mathcal{V}:=\mathbb{R}^{d}$ (regarded as a tychastic space of tyches);

2. a (single-valued) map $f: X \times \mathcal{V} \mapsto X$ defining the differential equation $x^{\prime}(t)=$ $f(x(t), v(t))$ parameterized by tyches $v$ (interpreted as a tychastic system);

3. a set-valued map $V: x \leadsto V(x)$ (interpreted as tychastic (set-valued) map);

4. a family $\widetilde{V}$ of tychastic retroactions $\widetilde{v}:(t, x): \mathbb{R}_{+} \times K \mapsto \widetilde{v}(t, x) \in V(x)$.

We associated with these data the set-valued map

$$
f_{[\widetilde{V}]}(t, x):=\bigcup_{\widetilde{v} \in \widetilde{V}} f(x, \widetilde{v}(t, x))
$$

and the tychastic system

$$
x^{\prime}(t) \in f_{[\widetilde{V}]}(t, x(t))
$$


It generates the evolutionary system $\mathcal{S}_{\widetilde{V}}: X \leadsto \mathcal{C}(0, \infty ; X)$ where $\mathcal{S}_{\widetilde{V}}(x)$ is the set of solutions $x(\cdot)$ of $x^{\prime}(t) \in f_{[\widetilde{V}]}(t, x(t))$ such that $x(0)=x$.

Let $\mathcal{H} \subset \mathcal{C}(0, \infty ; X)$ be a subset of evolutions, For example, subsets of evolutions

1. viable in the environment

$$
\mathcal{V}(K):=\{x(\cdot) \text { such that } \forall t \in[0, T], x(t) \in K\}
$$

2. capturing the target

$$
\mathcal{K}(K, C):=\{x(\cdot) \mid \exists T \geq 0 \text { such that } x(T) \in C \& \forall t \in[0, T], x(t) \in K\}
$$

3. viable in the environment until capturing the target

$$
\mathcal{V}(K, C):=\mathcal{V}(K) \cup \mathcal{K}(K, C)
$$

Let us consider a subset $\mathcal{H} \subset \mathcal{C}\left(0, \infty ; \mathbb{R}^{d}\right)$ of evolutions. The "core" $\mathcal{S} \mathcal{S}^{\ominus 1}(\mathcal{H})$ of $\mathcal{H}$ under set-valued $\operatorname{map} \mathcal{S}_{\widetilde{V}}$ is the set

$$
\mathcal{S}_{\widetilde{V}}^{\ominus 1}(\mathcal{H}):=\left\{x \in X \text { such that } \mathcal{S}_{\widetilde{V}}(x) \subset \mathcal{H}\right\}
$$

of initial states such that all evolutions starting from $x$ belong to $\mathcal{H}$ and share its properties.

We obtain the following concepts of invariance kernel of the environment with target

$$
\operatorname{Inv}_{f}([\widetilde{V}] ;(K, C)):=\mathcal{S}_{\widetilde{V}}^{\ominus 1}(\mathcal{V}(K, C))
$$

by taking the core under the tychastic system of the family of evolutions viable in the environment until capturing the target.

If we suppose that all evolutions starting from $K \backslash C:=K \cap \complement C$ leave $K \backslash C$ in finite time, all evolutions starting from the invariance kernel with target are viable in the environment until they capture the target.

By defining the order relation $V_{1} \subset V_{2}$ on set-valued maps by the inclusion relation $\operatorname{Graph}\left(V_{1}\right) \subset \operatorname{Graph}\left(V_{2}\right)$ on their graphs, we note that the application $(\widetilde{V}, K, C) \mapsto$ $\operatorname{Inv}_{f}([\tilde{V}] ;(K, C))$ is decreasing with respect to the tychastic map and increasing with respect to $K$ and $C$. It shows especially that the invariance kernel is the largest fixed point $G$ between $C$ and $K$ of the map $K \mapsto \operatorname{Inv}_{f}([\widetilde{V}] ;(K, C))$ :

$$
C \subset G \subset K \text { et } G=\operatorname{Inv}_{f}([\widetilde{V}] ;(G, C))
$$

as well as the unique fixed point $G$ between $C$ and $K$ of the map $C \mapsto \operatorname{Inv}_{f}([\widetilde{V}] ;(K, C))$ and thus the unique bilateral fixed point

$$
C \subset G \subset K \text { et } G=\operatorname{Inv}_{f}([\widetilde{V}] ;(G, C))=\operatorname{Inv}_{f}([\widetilde{V}] ;(K, G))
$$


The topological properties of invariance kernels (closure and stability of the basin and kernels, etc.) are proved under the assumption that the dynamics of $f$ and $V$ tychastic set-valued map are Lipschitz.

Viability and invariance theorems characterize the basin invariance kernel and invariance kernels by tangential properties at the boundary relating velocities and tangent directions to the environment.

\subsection{Impulse Tychastics Systems}

Consider a impulse tychastic system $(f(V]), \Phi)$ (where $V$ is a tychastic map identified with its graph $\operatorname{Graph}(V) \subset X \times \mathcal{V})$ and an environment.

Discontinuous evolutions (with "jumps") are governed by the impulse system if

$$
\begin{cases}(i) & x^{\prime}(t) \in f_{[\widetilde{V}]}(t, x(t)) \text { whenever } x(t) \in K \backslash \operatorname{Dom}(\Phi) \\ (i i) & x(t)=\Phi(x(t)) \text { whenever } x(t) \in \operatorname{Dom}(\Phi)\end{cases}
$$

In other words, all evolutions starting from the initial state $x \in \operatorname{Inv}_{f}([V] ;(K, \operatorname{Dom}(\Phi)))$ are viable in $K$ until they reaches at finite time $t^{\sharp}$ the domain $\operatorname{Dom}(\Phi)$ of the reset map $\Phi$ at a point $x\left({ }^{-} t^{\sharp}\right) \in \operatorname{Dom}(\Phi)$. The impulse system instantly resets this state at the new state $x\left(t^{\sharp}\right)=\Phi\left(x\left({ }^{-} t^{\sharp}\right)\right)$. If the image of $\Phi$ is contained $\operatorname{in}_{\operatorname{Inv}_{f}}([V] ;(K, \operatorname{Dom}(\Phi)))$, the same scenario starts again.

Impulse viability means that

$$
\operatorname{Im}(\Phi) \subset \operatorname{Inv}_{f}([V] ;(K, \operatorname{Dom}(\Phi)))
$$

If $C \subset K$ is a target (for instance, the domain $\operatorname{Dom}(\Phi)$ of the reset map), we set

$$
\operatorname{Inv}[V]:=\operatorname{Inv}_{f}([V] ;(K, C))
$$

The invariance operator Inv is the map $V: \mapsto \operatorname{Inv}[V]$.

The question is

$$
\text { Look for } V \text { such that } \operatorname{Im}(\Phi) \subset \operatorname{Inv}[V]
$$

and, even, whether there is a largest tychastic set-valued map $V$ solution of this problem. The answer is positive.

\subsection{Tychastic Reliability}

The operator of invariance satisfies the following properties :

1. If $V_{1} \subset V_{2}$, then $\operatorname{Inv}\left[V_{2}\right] \subset \operatorname{Inv}\left[V_{1}\right]$;

2. If $G \subset \operatorname{Inv}\left[V_{1}\right] \cap \operatorname{Inv}\left[V_{2}\right]$, then $G \subset \operatorname{Inv}\left[V_{1} \cup V_{2}\right]$. 
These are the two properties required to define the Galois transform of the invariance operator defined by

$$
\operatorname{Fiab}[G]:=\bigcap_{G \subset \operatorname{Inv}[V]} \operatorname{Graph}(V)
$$

We will call it for reasons that are obvious the reliability operator (for the French opérateur de fiabilité) Fiab mapping subsets $G$ to graphs of tychastic maps, subset of $X \times \mathcal{V}$.

The reliability operator verifies the properties

1. if $G_{1} \subset G_{2}$, then $\operatorname{Fiab}\left[G_{2}\right] \subset \operatorname{Fiab}\left[G_{1}\right]$;

2. If $V \subset \operatorname{Fiab}\left[G_{1}\right] \cap \operatorname{Fiab}\left[G_{2}\right]$, then $V \subset \operatorname{Fiab}\left[G_{1} \cup G_{2}\right]$.

These operators satisfy

$$
V \subset \operatorname{Fiab}[\operatorname{Inv}[V]] \text { and } G \subset \operatorname{Inv}[\operatorname{Fiab}[G]]
$$

The invariance operator is itself the Galois transform of the reliability operator

$$
\operatorname{Inv}[V]:=\bigcap_{G \subset \operatorname{Fiab}[G]} G
$$

We also note that the maps $V \mapsto \operatorname{Fiab}[\operatorname{Inv}[V]]$ and $G \subset \operatorname{Inv}[\operatorname{Fiab}[G]]$ are "closures" (increasing, extensive and idempotent applications). We refer to Chapter 7 of [4, Aubin] and for more details, [1, Akian, Gaubert \&Kolokotsov] and its bibliography for references ont this topic.

\subsection{Regulated Tychastic Systems}

We further introduces

1. a space $\mathcal{U}:=\mathbb{R}^{b}$ (interpreted as a control space or regulon space);

2. a map $f: X \times \mathcal{U} \times \mathcal{V} \mapsto X$ defining the differential equation $x^{\prime}(t)=f(x(t), u(t), v(t))$ parameterized by controls $u$ and tyches $v$ (interpreted as a controlled or regulated tychastic system);

3. a set-valued map $U: x \sim U(x)$ (interpreted as the contingent set-valued map );

4. a family $\widetilde{U}$ of contingent retroactions $\widetilde{u}:(t, x): \mathbb{R}_{+} \times K \mapsto \widetilde{u}(t, x) \in U(x)$.

We associate with these new data the set-valued map

$$
f_{[\widetilde{u}, \widetilde{V}]}(t, x):=f_{[\widetilde{V}]}(t, \widetilde{u}(t, x))
$$

and the controlled (or regulated) tychastic system

$$
x^{\prime}(t) \in f_{[\widetilde{V}]}(t, \widetilde{u}(t, x(t)))
$$


The guaranteed viability kernel is defined by

$$
\operatorname{GuarViab}_{f}([\widetilde{U}, \widetilde{V}] ; K, C):=\bigcup_{\widetilde{u} \in \widetilde{U}} \operatorname{Inv}_{f}([\widetilde{u}, \widetilde{V}] ; K, C)
$$

which depends on the set $K$ on one hand and on the pair $[\widetilde{U}, \widetilde{V}]$ made of retroactions $\widetilde{u} \in \widetilde{U}$ defining the contingent uncertainty and $\widetilde{v} \in \widetilde{V}$ defining the tychastic uncertainty, on the other hand.

A retroaction $u^{\curvearrowright} \in \widetilde{U}$ is a control (or management) rule if

$$
\operatorname{GuarViab}_{f}\left(\left[u^{\odot}, \widetilde{V}\right] ; K, C\right)=\operatorname{GuarViab}_{f}([\widetilde{U}, \widetilde{V}] ; K, C)
$$

Pierre Bernhard was one of the first to parameterize the concepts of values of differential games by families of retroactions $\widetilde{U}$ and $\widetilde{V}$ of the two players Ursula and Victor. It is this suggestion that we follow for defining the concept of guaranteed viability kernel in this "game against Nature" which is nothing else than a controlled tychastic system.

We note that the map

$$
(K, C, \widetilde{U}, \widetilde{V}) \mapsto \operatorname{GuarViab}_{f}([\widetilde{U}, \widetilde{V}] ; K, C)
$$

is increasing respect to $K, C$ and $\widetilde{U}$, on the one hand, and decreasing with respect to $\widetilde{V}$, on the other hand (for the inclusion relation sets).

If tychastic uncertainty (described by $\widetilde{V}$, and, upstream, by the graph of the tychastic set-valued map $V$ ) increases, the guaranteed viability kernel guaranteed decreases, so it is necessary to also increase the set $\widetilde{U}$, and, upstream, the graph of the contingent set-valued map $U$ (translating contingent uncertainty) for increasing the guaranteed viability kernel. Once the environment $K$ and $C$ the target are given, the map

$$
(\widetilde{U}, V) \mapsto \operatorname{GuarViab}_{f}([\widetilde{U}, \widetilde{V}] ; K, C)
$$

leads to a new concept of "game" on set-valued maps $U$ and $V$ and taking values in the family of (closed) subsets of the state space. In this context, we choose here the set $\widetilde{V}$ of open loop feedbacks and the set $\widetilde{U}$ a set of closed loop feedbacks. However, many other options have been proposed and studied.

Viability theory provides mathematical and algorithmic properties of guaranteed viability kernels , including the fixed point property of the guaranteed viability kernel guarantee, simple to state but important : the guaranteed viability kernel is the largest subset $G$ between $C$ and $K$ satisfying the fixed point property

$$
G=\operatorname{GuarViab}_{f}([\widetilde{U}, \widetilde{V}] ; G, C)
$$


The viability algorithms provide means to compute the guaranteed viability kernels and to program them (see Section 5, p. 25] and [52, Saint-Pierre], [40, Cardaliaguet, Quincampoix \& Saint-Pierre], among many other papers).

In the same way that it is impossible to summarize in few pages the properties of stochastic differential equations for a layman in probability, it is impossible to go beyond the basic concepts and technical viability, even briefly. This is a reason why we invite the reader who would like to deepen these results to refer to the literature on viability theory.

\subsection{Qualitative and quantitative Insurance Evaluations and Mea- sures}

The set of real numbers equipped with the usual ordering is the favorite candidate for providing measure processes of elements of a set $A$ by a function $\mathbf{a}: \mathcal{A} \mapsto \mathbb{R}$. This is the case of several families $\mathcal{A} \subset \mathcal{P}(E)$ of subsets of a space $E$. If $\mathcal{A}$ is a $\sigma$-algebra, the concept of Kolmogorov measure provides the best known example. If the set $\mathcal{A}$ is the set of compact subsets and if we equip $\mathbb{R}$ with the max-plus algebra (for the operations $\max (a, b)$ and $a+b$ ), Maslov measure $A \mapsto \sup _{x \in A} \mu(x)$ provides another example (The Cramer transform links those two examples). It is also in this context that one can define concepts similar to those of fuzzy sets to formulate mathematically other connotations of chance (see [11, Aubin \& Dordan]).

Actually, any map a from $\mathcal{A}$ to a lattice could provide evaluation and comparison procedures of its elements, but not a measure, since the meaning of "measure" generally involves the real numbers.

The concepts of viability theory (invariance kernels and guaranteed viability kernels with targets. etc.) are maps taking their values in the family of subsets, endowed with the inclusion order relation. Each of these applications may serve as an evaluation process. The guaranteed viability kernel provides a procedure for evaluating the concept of (tychastic) warranty (and thus, of its insurance), as large as the guaranteed viability kernel is small. This does not forbid, if necessary, to use these kernels and basins as "qualitative evaluations", first, and second, to use Kolmogorov, Maslov and other measurement procedures of subsets to further furnish a quantitative measure by numbers. This combination of qualitative and quantitative measures could offer meaningful and useful new instruments.

This is just the case of the initial minimum guaranteed investment we used in the VPPI approach of the ALM problem. 


\section{Stochastic and Tychastic Viability}

The invariance kernel is an example of the core $\mathcal{S}^{\ominus 1}(\mathcal{H})$ of a subset $\mathcal{H} \subset \mathcal{C}\left(0, \infty ; \mathbb{R}^{d}\right)$ for $\mathcal{H}=\mathcal{K}_{(K, C)}$ being the set of evolutions viable in $K$ reaching the target $C$ in finite time.

Let us consider random events $\omega \in \Omega$, where $(\Omega, \mathcal{F}, \mathbb{P})$ is a probability space, instead of tyches $v(\cdot) \in Q(x(\cdot))$.

A stochastic system is a specific parameterized evolutionary system described by a map $\mathbb{X}:(x, \omega) \in \mathbb{R}^{d} \times \Omega \mapsto \mathbb{X}(x, \omega) \in \mathcal{C}\left(0, \infty ; \mathbb{R}^{d}\right)$ (usually denoted by $t \mapsto \mathbb{X}_{\omega}^{x}$ in the stochastic literature) where $\mathcal{C}\left(0, \infty ; \mathbb{R}^{d}\right)$ is the space of continuous evolutions. In other words, it defines evolutions $t \mapsto \mathbb{X}(x, \omega)(t):=\mathbb{X}_{\omega}^{x}(t) \in \mathbb{R}^{d}$ starting at $x$ at time 0 and parameterized by random events $\omega \in \Omega$ satisfying technical requirements (measurability, filtration, etc.) that are not relevant to involve at this stage of the exposition. The initial state $x$ being fixed, the random variable $\omega \mapsto \mathbb{X}(x, \omega):=\mathbb{X}_{\omega}^{x}(\cdot) \in \mathcal{C}\left(0,+\infty ; \mathbb{R}^{d}\right)$ is called a stochastic process. A subset $\mathcal{H} \subset \mathcal{C}\left(0, \infty ; \mathbb{R}^{d}\right)$ of evolutions sharing a given property being chosen, it is natural, as we did for tychastic systems, to introduce the stochastic core of $\mathcal{H}$ under the stochastic system : it is the subset of initial states $x$ from which starts a stochastic process $\omega \mapsto \mathbb{X}(x, \omega)$ such that for almost all $\omega \in \Omega, \mathbb{X}(x, \omega) \in \mathcal{H}$ :

$$
\operatorname{Stoc}_{\mathbb{X}}(\mathcal{H}):=\left\{x \in \mathbb{R}^{d} \mid \text { for almost all } \omega \in \Omega, \mathbb{X}(x, \omega):=\mathbb{X}_{\omega}^{x}(\cdot) \in \mathcal{H}\right\}
$$

Regarding a stochastic process as a set-valued map $\mathbb{X}$ associating with any state $x$ the family $\mathbb{X}(x):=\{\mathbb{X}(x, \omega)\}_{\omega \in \Omega}$, the definitions of stochastic cores 32 of subsets of evolution properties are similar in spirit to definition :

$$
\mathcal{S}^{\ominus 1}(\mathcal{H}):=\left\{x \in \mathbb{R}^{d} \mid \text { for all } v(\cdot) \in Q(x(\cdot)), \quad x_{v(\cdot)}(\cdot) \in \mathcal{H}\right\}
$$

under a tychastic system

$$
x^{\prime}(t)=f(x(t), v(t)) \text { where } v(t) \in Q(x(t))
$$

Furthermore, the parameters $\omega$ are constant in the stochastic case, whereas the tychastic uncertainty $v(\cdot)$ is dynamic in nature, a more realistic assumption in the domain of life sciences. There is however a deeper similarity that we mention briefly. When the stochastic system $(x, \omega) \mapsto \mathbb{X}(x, \omega)$ is derived from a stochastic differential equation, the Strook-Varadhan Support Theorem (see [53, 54, Stroock \& Varadhan]) states that there exists a tychastic system $(x, v) \mapsto \mathcal{S}(x, v)$ such that, whenever $\mathcal{H}$ is closed, the stochastic core of $\mathcal{H}$ under the stochastic system $\mathbb{X}$ and its tychastic core under the associated tychastic system $\mathcal{S}$ coincide :

$$
\operatorname{Stoc}_{\mathbb{X}}(\mathcal{H})=\mathcal{S}^{\ominus 1}(\mathcal{H})
$$

To be more specific, let $\mathbb{X}(x, \omega)$ denote the solution starting at $x$ to the stochastic differential equation

$$
d x=\gamma(x) d t+\sigma(x) d W(t)
$$


where $W(t)$ ranges over $\mathbb{R}^{c}$ and the drift $\gamma: \mathbb{R}^{d} \mapsto \mathbb{R}^{d}$ and the diffusion $\sigma: \mathbb{R}^{d} \mapsto \mathcal{L}\left(\mathbb{R}^{c}, \mathbb{R}^{d}\right)$ are smooth and bounded maps. Let us associate with them the Stratonovitch drift $\widehat{\gamma}$ defined by $\widehat{\gamma}(x):=\gamma(x)-\frac{1}{2} \sigma^{\prime}(x) \sigma(x)$. The Stratonovitch stochastic integral is an alternative to the Ito integral, and easier to manipulate. Unlike the Ito calculus, Stratonovich integrals are defined such that the chain rule of ordinary calculus holds. It is possible to convert Ito stochastic differential equations to Stratonovich ones.

Then, the associated tychastic system is

$$
x^{\prime}(t)=\widehat{\gamma}(x(t))+\sigma(x(t)) v(t) \text { where } v(t) \in \mathbb{R}^{c}
$$

where the tychastic map is constant and equal to $\mathbb{R}^{c}$.

Consequently, the tychastic system associated with a stochastic one by the StrookVaradhan Support Theorem is very restricted : there are no bounds at all on the tyches, whereas general tychastic systems allow the tyches to range over subsets $Q(x)$ depending upon the state $x$, describing so to speak a state-dependent uncertainty :

$$
x^{\prime}(t)=\widehat{\gamma}(x(t))+\sigma(x(t)) v(t) \text { where } v(t) \in Q(x(t))
$$

This state-dependent uncertainty, unfortunately absent in the mathematical representation of uncertainty in the framework of stochastic processes, is of utmost importance for describing uncertainty in problems dealing with living beings.

When $\mathcal{H}$ is a Borelian of $\mathcal{C}\left(0, \infty ; \mathbb{R}^{d}\right)$, we denote by $\mathbb{P}_{\mathbb{X}(x, \cdot)}$ the law of the random variable $\mathbb{X}(x, \cdot)$ defined by

$$
\mathbb{P}_{\mathbb{X}(x, \cdot)}(\mathcal{H}):=\mathbb{P}(\{\omega \mid \mathbb{X}(x, \omega) \in \mathcal{H}\})
$$

Therefore, we can reformulate the definition of the stochastic core of a set $\mathcal{H}$ of evolutions in the form

$$
\operatorname{Stoc}_{\mathbb{X}}(\mathcal{H})=\left\{x \in \mathbb{R}^{d} \mid \mathbb{P}_{\mathbb{X}(x, \cdot)}(\mathcal{H})=1\right\}
$$

In other words, the stochastic core of $\mathcal{H}$ is the set of initial states $x$ such that the subset $\mathcal{H}$ has probability one under the law of the stochastic process $\omega \mapsto \mathbb{X}(x, \omega) \in \mathcal{C}\left(0,+\infty ; \mathbb{R}^{d}\right)$ (if $\mathcal{H}$ is closed, $\mathcal{H}$ is called the support of the law $\mathbb{P}_{\mathbb{X}(x, \cdot)}$ ). The Strook-Varadhan Support Theorem states that under regularity assumptions, this support is the core of $\mathcal{H}$ under the tychastic system (33). See [45, Doss] and [12, Aubin\& Doss] for more details. It furthermore provides a characterization of stochastic viability in terms of tangent cones and general curvatures of the environments. Characterization of stochastic viability and invariance in terms of stochastic tangent cones has been carried over in [8, 9, Aubin \& Da Prato] and [10, Aubin, Da Prato\& Frankowska] and [42, 43, 44, Da Prato \& Frankowska] in terms of distance functions among many other studies in this direction.

These remarks further justify our choice of privileging tychastic systems because, as far as the properties of initial states of evolution are concerned, stochastic systems are just (very) particular cases of tychastic systems. 
Acknowledgements The authors thank Pierre Bernhard, Pierre Cardaliaguet and Marc Quincampoix for their contributions in differentiable game theory, Giuseppe Da Prato, Halim Doss, Hélène Frankowska and Jerzy Zabczyk for dealing with stochastic and tychastic viability, Daniel Gabay, Nadia Lericolais, Domique Pujal and Francine Roure for their initiation to finance, Marie-Hélène Durand regarding the uncertainty and Sophie Martin concerning resilience and other tychastic indicators. 


\section{Références}

[1] Akian M, Gaubert S. and Kolokotsov V. (2005) Set Coverings and Invertibility of Functional Galois Connections, Contemporary Mathematics, 377, 19-51

[2] Aubin J.P. (1991) Viability Theory, Birkhäuser

[3] Aubin, J.-P. (1997). Dynamic Economic Theory : A Viability Approach. Springer-Verlag.

[4] Aubin J.-P. (2000) Mutational and morphological analysis : tools for shape regulation and morphogenesis, Birkh'auser

[5] Aubin J.-P. (2000) Optimal impulse control problems and quasi-variational inequalities thirty years later : a viability approach, in Optimal Control and Partial Differential Equations, 311-324, IOS Press

[6] Aubin J.-P. (2010) La mort du devin, l'émergence du démiurge. Essai sur la contingence et la viabilité des systèmes, Éditions Beauchesne

[7] Aubin J.-P., Bayen A. and Saint-Pierre P. (2010) Viability Theory Twenty Years Later. Regulation of Uncertain Systems, Springer-Verlag

[8] Aubin J.-P. \& Da Prato G. (1995) Stochastic Nagumo's Viability Theorem, Stochastic Analysis and Applications, 13, 1-11

[9] Aubin J.-P. \& Da Prato G. (1998) The Viability Theorem for Stochastic Differential Inclusions, Stochastic Analysis and Applications, 16, 1-15

[10] Aubin J.-P., Da Prato G. \& Frankowska H. (2000) Stochastic invariance for differential inclusions, J. Set-Valued Analysis, 8, 181-201

[11] Aubin J.-P. \& Dordan O. (1996) Fuzzy Systems, Viability Theory and Toll Sets, In Handbook of Fuzzy Systems, Modeling and Control, Hung Nguyen Ed.. Kluwer, 461-488

[12] Aubin J.-P. \& Doss H. (2003) Characterization of Stochastic Viability of any Nonsmooth Set Involving its Generalized Contingent Curvature, Stochastic Analysis and Applications, 25, 951-981

[13] Aubin J.-P and Frankowska H. (1990), Set-Valued Analysis, Birkh'auser Boston, Boston,

[14] Aubin J.-P. \& Haddad G. (2001) Path-dependent impulse and hybrid control systems, in Hybrid Systems : Computation and Control, 119-132, Di Benedetto \& SangiovanniVincentelli Eds, Proceedings of the HSCC 2001 Conference, LNCS 2034, Springer-Verlag

[15] Aubin J.-P. \& Haddad G. (2002) History (Path) Dependent Optimal Control and Portfolio Valuation and Management, J. Positivity, 6, 331-358

[16] Aubin J.-P. \& Haddad G. (2001) Cadenced Runs of Impulse and Hybrid Control Systems, International Journal Robust and Nonlinear Control, 11, 401-415

[17] Aubin J.-P., Lygeros J., Quincampoix M., Sastry S. \& Seube N. (2002) Impulse Differential Inclusions : A Viability Approach to Hybrid Systems, IEEE Transactions on Automatic Control, 47, 2-20 
[18] Aubin J.-P., Pujal D. \& Saint-Pierre P. (2005) Dynamic Management of Portfolios with Transaction Costs under Tychastic Uncertainty, in Numerical Methods in Finance, BENAmeur H. \& Breton M. Eds., Kl'uwer

[19] Aubin J.-P. \& Saint-Pierre P. (2006) A Tychastic Approach to Guaranteed Pricing and Management of Portfolios under Transaction Constraints, in Proceedings of the 2005 Ascona Conferences on Stochastic Analysis, Random Fields and Applications, Progress in Probability' of Birkh'auser Verlag

[20] Benaïm M. and El Karoui N. (2005) Promenade aléatoire : Chaînes de Markov et simulations; martingales et stratégies, Ecole Polytechnique

[21] Bensoussan A. (1984) On the Theory of Option Pricing, J. Acta Applicandae Mathematicae

[22] Bensoussan A. (1985) Concept for Risky Venture, Optimal Control Theory and Economic Analysis, 2

[23] Bensoussan A. ( 2000) Quelques remarques sur le prix des options avec prise en compte de contraintes, dans Décision Prospective Auto-organisation, Mélanges en l'honneur de J. Lesourne, eds J. Thépot, M. Godet, F. Roubelat, A.E. Saab, Dunod

[24] Bensoussan A. ( 2004) Remarks on the Pricing of Contingent Claims under Constraints, IEEE Transactions on Automatic Control, 49

[25] Bensoussan A. ( 2007) Real Options and Variational Inequalities, Proceedings of 46th IEEE Conference on Decision and Control, 12-14,

[26] Bensoussan A. ( 2008) Real Options, in Mathematical Modelling and Numerical Methods in Finance, edited by A. Bensoussan and Q. Zhang, Elsevier

[27] Bensoussan A. Crouhy M. and Galai D. (1994) Stochastic equity volatility related to the leverage effect, Applied Mathematical Finance 1, 63-85

[28] Bensoussan A. and Bernhard P. (1992) Remarks on the Theory of Robust Control, International Series of Numerical mathematics, Vol 107 Birkhauser

[29] Bensoussan A. Crouhy M. and Galai D. (1994) Stochastic equity volatility and the capital structure of the firm, The Royal Society, Phil.Trans.R. Soc.Lond. A 347

[30] Bensoussan A. Crouhy M. and Galai D. (1995) Stochastic volatility related to the leverage effect II : Valuation of European equity options and warrants, Applied Mathematical Finance, 2, 43-60

[31] Bensoussan A. Crouhy M. and Galai D. (1995) Black Scholes approximation of warrant prices, colloque Finance, 1992, ed. M. Jeanblanc-Pique, Futures and Options Research, Vol. 8, 1-14, JAI Press

[32] Bensoussan A. and Julien H. (1999) Option Pricing in a Market with frictions in Stochastic Analysis, Control, Optimization an Applications, Volume in honor of Professor W. Fleming, Systems and Control Foundations and Applications, W. Mc Eneany and al eds, Birkhauser, 521-540 
[33] Bensoussan A. and Julien H. (2000) On the pricing of contingent claims with frictions, J. Mathematical Finance, 10, 89 -108

[34] Bensoussan A.. Keppo J. and Sethi S.P. (2009) Optimal Consumption and Portfolio Decisions with Partially Observable Real Prices, Mathematical Finance

[35] Bensoussan A. and Lions J.L. (1982) Impulse Control and Quasi-Variational Inequalities, Dunod

[36] Bensoussan A., Menaldi J.M. (1997) Hybrid Control and Dynamic Programming, Dynamics of Continuous, Discrete and Impulsive Systems, 3

[37] Bensoussan A., Menaldi J.M. and Touzo N. (2005) Penalty Approximation and Analytical Characterization of the Problem of Super-replication Under Portfolio Constraints, Journal Asymptotic Analysis 42, 113-330, 2005

[38] Bouchaud J.-P. and Potters M. (2009) Theory of Financial Risk and Derivative Pricing: From Statistical Physics to Risk Management, Cambridge University Press

[39] Boulier J.-F. and Kanniganti A. (2005) Expected Performance and Risk of Various Portfolio insurance strategies, http ://www.actuaries.org/AFIR/colloquia/Brussels/BoulierKanniganti.pdf, May

[40] Cardaliaguet P., Quincampoix M. \& Saint-Pierre P. (1999) Set-valued numerical methods for optimal control and differential games, In Stochastic and differential games. Theory and numerical methods, Annals of the International Society of Dynamical Games, 177-247 Birkh'auser

[41] Crouhy M., Galai D. \& Mark R. (2006) The essentials of Risk Management McGrawHill

[42] Da Prato \& Frankowska (1994) A stochastic Filippov Theorem, Stochastic Calculus, 12, $409-426$

[43] Da Prato \& Frankowska (2001) Stochastic viability for compact sets in terms of the distance function, Dynamics Systems Appl., 10, 177-184

[44] Da Prato \& Frankowska (2004) Invariance of stochastic control systems with deterministic arguments,

[45] Doss H. (1977) Liens entre équations différentielles stochastiques et ordinaires, Ann. Inst. Henri Poincaré, Calcul des Probabilités et Statistique, 23, 99-125

[46] Frankowska H. (1991) Lower semicontinuous solutions to Hamilton-Jacobi-Bellman equations, in Proceedings of the 30th IEEE Conference on Decision and Control, Brighton, UK,

[47] Frankowska H. (2010) Control under state constraints, Proceedeings of the International Congress of Mathematicians (ICM 2010), Hyderabad, India

[48] Neftci S. N. (2009) Principles of Financial Engineering Academic Press 
[49] Planchet F. and Therond P. (2007) Mesure et gestion des risques d'assurance : Analyse critique des futurs référentiels prudentiel et d'information financière Economica [49, Planchet \&Therond] and [50, Planchet, Kamega A. \&Therond]

[50] Planchet F., Kamega A. and Therond P. (2009)Scenarios Economiques en AssuranceModélisation et Simulation Economica

[51] Rockafellar R.T. \& Wets R. (1997) Variational Analysis, Springer-Verlag

[52] Saint-Pierre P. (1994) Approximation of the viability kernel, Applied Mathematics \& Optimisation, 29, 187-209

[53] Stroock D.W. \& Varadhan S.R.S. (1972) On the support of diffusion processes with applications to the strong maximum principle, Proc. of the sixth Berkeley Symposium on mathematical statistics and Probabilty, Univ. of California Press, Vol. III. Probability Theory, 333-359

[54] Stroock D.W. \& Varadhan S.R.S. (1979) Multidimensional diffusion processes, SpringerVerlag

[55] Zabczyk J. (1996) Chance and decision : stochastic control in discrete time, Quaderni, Scuola Normale di Pisa 


\section{Table des matières}

$1 \quad$ Asset-Liability Management : the Insurance Problem 4

1.1 Insuring Liabilities . . . . . . . . . . . . . . . . . . . . . . . . . . 4

1.2 Assets portfolios and their exposure . . . . . . . . . . . . . . . . . . 5

1.3 Portfolio insurance : a viability problem . . . . . . . . . . . . . . 7

\begin{tabular}{llr}
\hline 2 & Uncertainties & 7
\end{tabular}

2.1 Stochastic Uncertainty . . . . . . . . . . . . . . . . . . . . . . . . . . . . . . . . . . . . . . .

2.2 Tychastic Uncertainty . . . . . . . . . . . . . . . . . . . . . . . . . 9

2.3 Nature and Measures of Insurance . . . . . . . . . . . . . . . . . . . . . . . 10

2.4 Contingent Uncertainty . . . . . . . . . . . . . . . . . . . . . 10

3 Predictive and Impulse Approaches 13

3.1 Predictive Approach . . . . . . . . . . . . . . . . . . . . . . . 13

3.2 Impulse Approach. . . . . . . . . . . . . . . . . . . . . . . . . 16

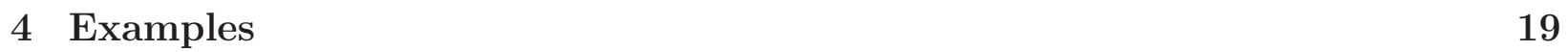

4.1 Predictive Case . . . . . . . . . . . . . . . . . . . . . . . . . . 19

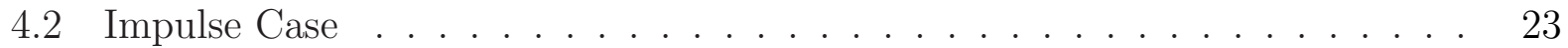

5 The VPPI and FPPI management software 25

5.1 Flow Chart of the VPPI Software . . . . . . . . . . . . . . . . . . . 26

5.2 Flow Chart of the FPPI Software . . . . . . . . . . . . . . . . . . . 27

\begin{tabular}{lll}
\hline 6 & Guaranteed Viability Kernels & 27
\end{tabular}

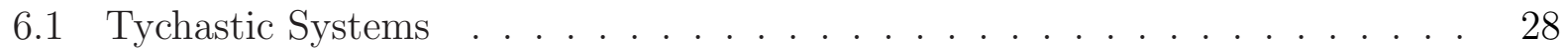

6.2 Impulse Tychastics Systems . . . . . . . . . . . . . . . . . . . . . . . . . . . . . . . . . . . . . . . . . . .

6.3 Tychastic Reliability . . . . . . . . . . . . . . . . . . . . . . . 30

6.4 Regulated Tychastic Systems _. . . . . . . . . . . . . . . . . 31

6.5 Qualitative and quantitative Insurance Evaluations and Measures . . . . . . 33

\begin{tabular}{|lll}
\hline 7 & Stochastic and Tychastic Viability & 34 \\
\hline
\end{tabular} 